\title{
Removing SPR-like Conditions in Adaptive Feedforward Control of Uncertain Systems
}

\author{
Yang Wang, Gilberto Pin, Andrea Serrani and Thomas Parisini
}

\begin{abstract}
The paper considers the problem of designing Adaptive Feedforward Control (AFC) systems for uncertain SISO linear systems perturbed by multi-sinusoidal disturbances of known frequencies. The proposed approach removes the longstanding assumption that either the sign of the real part or the imaginary part of the transfer function of a stable plant at the frequency of excitation be known for AFC to be applicable, which is referred to in this paper as an SPR-like condition. Notable features of the solution are that persistence of excitation is not required and stability analysis tools based on averaging are avoided; hence, the requirement of an exponentially stable equilibrium for the closed-loop system is circumvented.
\end{abstract}

\section{Introduction And Problem Statement}

In this paper, we consider the classic setup of the Adaptive Feedforward Control (AFC) problem for uncertain SISO LTI systems, shown in Figure 1 (see [1], [2]), where

$$
\begin{aligned}
\dot{x} & =A(\mu) x+B(\mu)[\hat{d}(t)-d(t)], \quad x(0)=x_{0} \in \mathbb{R}^{n_{0}} \\
y & =C(\mu) x
\end{aligned}
$$

is the realization of the internally stable interconnection of an uncertain plant model and a robust stabilizer. The vector $\mu \in \mathbb{R}^{p}$ collects the uncertain parameters of the plant model, assumed to range over a given known compact set, $\mathcal{P} \subset \mathbb{R}^{p}$. For future use, we let $W(s):=C(\mu)(s I-A(\mu))^{-1} B(\mu)$ denote the transfer function of System (1). The input of this system is affected by the sinusoidal disturbance

$$
d(t)=\sum_{n \in \mathcal{N}}\left[\psi_{n, 1} \cos \left(\omega_{n} t\right)+\psi_{n, 2} \sin \left(\omega_{n} t\right)\right]
$$

comprising $N$ known distinct excitation frequencies $\omega_{n} \in$ $\mathbb{R}_{>0}$ and uncertain parameters $\psi_{n}:=\left(\psi_{n, 1} \psi_{n, 2}\right)^{\top} \in$ $\mathbb{R}^{2}, n \in \mathcal{N}:=\{1,2, \cdots, N\}$. The disturbance input is matched with a disturbance estimate $\hat{d}(t) \in \mathbb{R}$, generated by the Adaptive Feedforward Control module in Figure 1. In qualitative terms, the control objective is posed as finding the estimate $\hat{d}(\cdot)$ such that boundedness of all internal trajectories is maintained and the output is regulated to zero. The

This work has been partially supported by the European Union's Horizon 2020 Research and Innovation Programme under grant agreement No 739551 (KIOS CoE).

Yang Wang is with the Dept. of Electrical and Electronic Engineering at Imperial College London, UK. (yang.wang13@imperial.ac.uk)

Gilberto Pin is with Electrolux Italia S.p.A, Italy. (gilbertopinealice.it).

Andrea Serrani is with the Department of Electrical and Computer Engineering, The Ohio State University, Columbus OH, USA.

Thomas Parisini is with the Dept. of Electrical and Electronic Engineering at Imperial College London, UK, with the KIOS Research and Innovation Centre of Excellence, University of Cyprus, and with the Dept. of Engineering and Architecture at University of Trieste, Italy. (t.parisini@gmail.com)

Corresponding author: A. Serrani, serrani.1@osu.edu

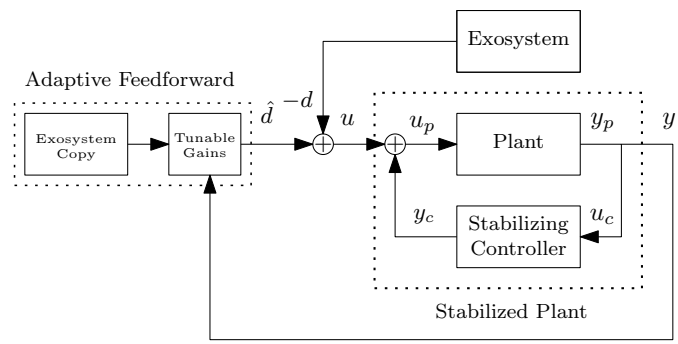

Fig. 1. Typical setup of the AFC problem

significance of this setup has been demonstrated in myriad applications to active noise control [3], helicopters [4], [5], disk drives [6], marine systems [7] and vibration control [8], to name but a few.

The standard continuous-time AFC algorithm of [1] requires $b_{n}:=\operatorname{sign} \operatorname{Re}\left\{W\left(j \omega_{n}\right)\right\}, n \in \mathcal{N}$, to be known a priori. In that case, the AFC module is comprised of the certainty-equivalence estimators

$$
\begin{aligned}
\dot{\hat{\psi}}_{n} & =-\varepsilon_{n} b_{n} \mathrm{e}^{S_{n}^{\top} t} g y, & & \hat{\psi}_{n}(0)=\hat{\psi}_{n 0} \in \mathbb{R}^{2} \\
\hat{d} & =\sum_{n=1}^{N} g^{\top} \mathrm{e}^{S_{n} t} \hat{\psi}_{n}, & & n \in \mathcal{N}
\end{aligned}
$$

where the matrices $S_{n}, g$ are defined as

$$
S_{n}=\left(\begin{array}{cc}
0 & \omega_{n} \\
-\omega_{n} & 0
\end{array}\right), \quad g=\left(\begin{array}{l}
1 \\
0
\end{array}\right)
$$

and $\varepsilon_{n}>0$ are gain parameters. Exponential stability of the interconnection (1)-(3) is proved to hold for sufficiently small values of $\varepsilon_{n}$ by way of averaging arguments [9], [10]. Persistence of the sign of $\operatorname{Re}\left\{W\left(j \omega_{n}\right)\right\}$ over the range of frequencies of interest is referred to as an SPR-like condition. A similar result is obtained in [11] using a time-invariant realization of (3). Therein, it is shown that, in case the quantity $\operatorname{sign} \operatorname{Im}\left\{W\left(j \omega_{n}\right)\right\}$ is known in place of $\operatorname{sign} \operatorname{Re}\left\{W\left(j \omega_{n}\right)\right\}$, an equivalent controller is obtained by replacing $g$ in (4) with $g=\left(\begin{array}{ll}0 & 1\end{array}\right)^{\top}$ and by letting $b_{n}:=-\operatorname{sign} \operatorname{Im}\left\{W\left(j \omega_{n}\right)\right\}$. The extension of [11] to the case of uncertain frequencies of excitation is proposed in [12]. The case of input delay is considered in [13], under the assumption of knowledge of the sign of the high-frequency gain.

For uncertain plant models, in absence of the crucial information on both the sign of the real and the imaginary parts of $W\left(j \omega_{n}\right)$, none of those strategies can be implemented. An adaptive solution that estimates $\operatorname{Re}\left\{W\left(j \omega_{n}\right)\right\}$ and $\operatorname{Im}\left\{W\left(j \omega_{n}\right)\right\}$ alongside $\psi_{n}$ was pursued in [14]. The 


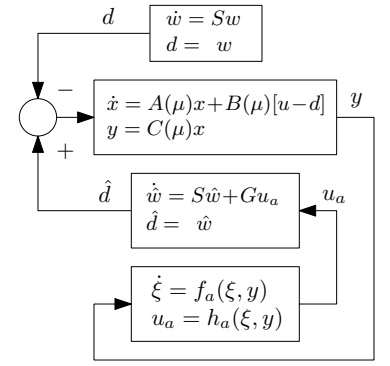

(a) Closed-loop system (5)-(9)

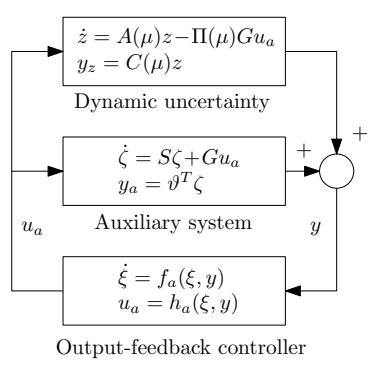

(b) Closed-loop system (8)-(9)
Fig. 2. Original closed-loop system (5)-(9) as the interconnection (8)-(9) of a dynamic uncertainty, an auxiliary system and a dynamic controller.

effectiveness of the scheme was demonstrated in simulations and experiments; however, the convergence properties of the algorithm could not be formally proved due to the absence of exponential stability, which is required for asymptotic analysis based on averaging, and local stability of the closedloop system could only be postulated. Furthermore, the issue of non-singularity of the control law, which requires bounding away from zero the euclidean norm of the estimates of $\operatorname{Re}\left\{W\left(j \omega_{n}\right)\right\}$ and $\operatorname{Im}\left\{W\left(j \omega_{n}\right)\right\}$, was not addressed. In [5], [15], a recursive least-squares estimator was employed for on-line identification of the frequency response of the plant. Therein, the analysis assumed that the plant model is in steady-state, hence the dynamic interaction between the AFC algorithm and the plant dynamics was neglected. The same type of assumption was made for the adaptive solution proposed in [16]. Robustness with respect to unstructured dynamic uncertainty acting on a known nominal plant model was considered in [17], [18].

In this paper, building upon the preliminary work [19], we propose an AFC strategy that, by disposing of the knowledge of both sign $\operatorname{Re}\left\{W\left(j \omega_{n}\right)\right\}$ and $\operatorname{sign} \operatorname{Im}\left\{W\left(j \omega_{n}\right)\right\}$, avoids the need for SPR-like conditions, which are replaced by a weaker observability condition for the disturbance. As in the cited references, the plant model is unknown, but assumed to be robustly stable. The solution makes use of tools from direct adaptive control [20], multi-parametric estimation [21]-[25] and $\mathcal{L}_{2}$-gain analysis [26]. To the best of our knowledge, this is the first result that provides asymptotic regulation in the presence of parametric model uncertainty where the dynamics of the plant is explicitly accounted for in the stability analysis.

Notation: Throughout the paper, $\|\cdot\|$ denotes Euclidean norms of vectors and matrices, whereas $\|\cdot\|_{\mathcal{L}_{2}}$ denotes norms in $\mathcal{L}_{2} . I$ denotes the generic identity matrix. For vectors $x_{i} \in$ $\mathbb{R}^{n}, \operatorname{col}\left(x_{1}, \ldots, x_{N}\right):=\left(x_{1}^{\top} \cdots x_{N}^{\top}\right)^{\top} \in \mathbb{R}^{n N}$. For vectors or matrices $A_{i} \in \mathbb{R}^{m \times n}, \oplus_{i=1}^{N} A_{i}:=\operatorname{diag}\left(A_{1}, \ldots, A_{N}\right) \in$ $\mathbb{R}^{m N \times n N}$. For a set $\mathcal{A} \subset \mathbb{R}^{n}, \mathcal{A}^{N}:=\mathcal{A} \times \cdots \times \mathcal{A}$.

\section{A. Statement of the Problem}

We begin by replacing in (3) the feedback terms $-\varepsilon_{n} b_{n} y$ with auxiliary inputs, $u_{a n}$, to be defined. A coordinate change is then applied to dispose of the need to consider a time-varying setting. As in [11], define $w_{n}(t):=$ $\exp \left(S_{n} t\right) \psi_{n}$ and apply the transformation $\hat{w}_{n}(t):=$ $\exp \left(S_{n} t\right) \hat{\psi}_{n}$ to rewrite (1)-(3) as the interconnection of an exosystem, an uncertain internally stable plant, and a disturbance model (all linear and time-invariant) as follows:

$$
\begin{aligned}
& \dot{w}=S w, \quad w(0)=w_{0} \in \mathbb{R}^{2 N} \\
& \dot{x}=A(\mu) x+B(\mu)[\Gamma \hat{w}-\Gamma w], \quad x(0)=x_{0} \in \mathbb{R}^{n_{0}} \\
& \dot{\hat{w}}=S \hat{w}+G u_{a}, \quad \hat{w}(0)=\hat{w}_{0} \in \mathbb{R}^{2 N} \\
& y=C(\mu) x
\end{aligned}
$$

with $w:=\operatorname{col}\left(w_{1}, \ldots, w_{N}\right), \hat{w}:=\operatorname{col}\left(\hat{w}_{1}, \ldots, \hat{w}_{N}\right) \in \mathbb{R}^{2 N}$, $u_{a}:=\operatorname{col}\left(u_{a 1}, \ldots, u_{a N}\right) \in \mathbb{R}^{N}, S:=\operatorname{diag}\left(S_{1}, \cdots, S_{N}\right) \in$ $\mathbb{R}^{2 N \times 2 N}, G:=\operatorname{diag}(g, \cdots, g) \in \mathbb{R}^{2 N \times N}$ and $\Gamma:=$ $\left(g^{\top} \cdots g^{\top}\right) \in \mathbb{R}^{2 N}$.

Next, we clarify the statement that System (1) is internally stable, robustly in $\mu \in \mathcal{P}$ (see [27]):

Assumption 1.1: There exist constants $\varrho_{1}, \varrho_{2}>0$ such that the parameterized family $P_{z}: \mathbb{R}^{p} \rightarrow \mathbb{R}^{n \times n}$ of solutions of the Lyapunov equation $P_{z}(\mu) A(\mu)+A^{\top}(\mu) P_{z}(\mu)=-I$ satisfies $\varrho_{1} I \leq P_{z}(\mu) \leq \varrho_{2} I$ for all $\mu \in \mathcal{P}$.

Owing to Assumption 1.1, let $\Pi_{n}(\mu) \in \mathbb{R}^{n_{0} \times 2}$ denote the unique solution of the Sylvester equation $\Pi_{n}(\mu) S_{n}=$ $A(\mu) \Pi_{n}(\mu)+B(\mu) g^{\top}$, and define the unknown parameter vectors

$$
\vartheta_{n}^{\top}:=C(\mu) \Pi_{n}(\mu)=\left(\operatorname{Re}\left\{W\left(j \omega_{n}\right)\right\} \operatorname{Im}\left\{W\left(j \omega_{n}\right)\right\}\right)
$$

for $n \in \mathcal{N}$. Note that $\vartheta_{n}$ are functions of $\mu$, but this dependence will not be displayed for ease of notation. Set $\Pi(\mu):=\left(\Pi_{1}(\mu) \cdots \Pi_{N}(\mu)\right) \in \mathbb{R}^{n_{0} \times 2 N}$ and $\vartheta:=$ $\operatorname{col}\left(\vartheta_{1}, \ldots, \vartheta_{N}\right) \in \mathbb{R}^{2 N}$. Let the compact set $\Theta \subset \mathbb{R}^{2}$ be defined as the annular region

$$
\Theta:=\left\{\theta \in \mathbb{R}^{2} \mid \delta_{1}^{2} \leq\|\theta\|^{2} \leq \delta_{2}^{2}\right\}
$$

for given real numbers $0<\delta_{1}<\delta_{2}$. The SPR-like assumptions are replaced by the following weaker condition, which is independent on the sign of the entries of $\vartheta_{n}$ :

Assumption 1.2: The vectors $\vartheta_{n}, n \in \mathcal{N}$, satisfy $\vartheta_{n} \in \Theta$ for all $\mu \in \mathcal{P}$, that is, $\vartheta \in \Theta^{N}$.

Assumption 1.2 implies observability of the pair $\left(\vartheta_{n}, S_{n}\right)$, uniformly in $\mu \in \mathcal{P}$. Changing again variables as $\zeta:=\hat{w}-w$ and $z:=x-\Pi(\mu) \zeta$, one obtains the error system

$$
\begin{aligned}
& \dot{z}=A(\mu) z-\Pi(\mu) G u_{a}, \quad z(0)=z_{0} \in \mathbb{R}^{n_{0}} \\
& \dot{\zeta}=S \zeta+G u_{a}, \quad \zeta(0)=\zeta_{0} \in \mathbb{R}^{2 N} \\
& y=C(\mu) z+\vartheta^{\top} \zeta .
\end{aligned}
$$

The control problem can now be formally stated as follows:

Problem 1: Design a dynamic output-feedback controller

$$
\begin{aligned}
& \dot{\xi}=f_{a}(\xi, y), \quad \xi(0)=\xi_{0} \in \mathbb{R}^{m} \\
& u_{a}=h_{a}(\xi, y)
\end{aligned}
$$

such that all trajectories of the closed-loop System (8)-(9) originating from initial conditions $z_{0} \in \mathbb{R}^{n_{0}}, \zeta_{0} \in \mathbb{R}^{2 N}$ and $\xi_{0} \in \mathcal{X}$, where $\mathcal{X} \subset \mathbb{R}^{m}$ is a set to be determined, are bounded and satisfy $\lim _{t \rightarrow \infty} y(t)=0$ for all $\mu \in \mathcal{P}$. 
The following strategy is adopted to solve Problem 1: System (8)-(9) is regarded as the interconnection of an auxiliary system (the $\zeta$-dynamics), a dynamic perturbation (the $z$-dynamics), and a controller to be determined, as seen in Fig. 2(b). For the auxiliary system, a baseline adaptive controller is designed, and a suitable non-minimal realization of the observer error dynamics is derived, which is amenable to parameter estimation. This is accomplished in Section II. A stability analysis for the baseline controller is carried out in Section III with the purpose of determining the properties of the update law required to achieve asymptotic regulation. The necessity of constraining the parameter estimates in the non-convex parameter set $\Theta^{N}$ necessitates replacing the baseline controller with a multiple-model switching controller, presented in Section IV. The stability analysis of the overall system is presented in Section V, where global boundedness of closed-loop trajectories and asymptotic regulation are proved. A lower-dimensional implementation of the controller is suggested in Section VI. Finally, a detailed simulation study is presented in Section VII.

\section{Baseline Controller Design}

As the useful information for reconstructing $d(t)$ is the steady-state response $y_{\mathrm{ss}}(t)=\vartheta^{\top} \zeta(t)$ corresponding to $u_{a}=$ 0 and $\zeta_{0}=-w_{0}$, we associate to (8) the auxiliary system

$$
\begin{aligned}
& \dot{\zeta}=S \zeta+G u_{a} \\
& y_{a}=\vartheta^{\top} \zeta
\end{aligned}
$$

which is controllable and observable for all $\mu \in \mathcal{P}$, owing to Assumption 1.2. Note that the signal $y_{a}=y-C(\mu) z$ is not available for feedback. To design a dynamic controller for (10), we first obtain a more suitable realization by means of the coordinate transformations $\zeta_{o n}=M_{o n}^{-1} \zeta_{n}$, where

$$
M_{o n}:=\frac{1}{\left\|\vartheta_{n}\right\|^{2}}\left(\begin{array}{cc}
\vartheta_{n 1} & -\vartheta_{n 2} \\
\vartheta_{n 2} & \vartheta_{n 1}
\end{array}\right), \quad n \in \mathcal{N} .
$$

This yields the system

$$
\begin{aligned}
& \dot{\zeta}_{o}=S \zeta_{o}+\boldsymbol{\theta} u_{a} \\
& y_{a}=\Gamma \zeta_{o}
\end{aligned}
$$

where $\boldsymbol{\theta}:=\oplus_{n=1}^{N} \theta_{n} \in \mathbb{R}^{2 N \times N}, \theta_{n}=\left(\vartheta_{n 1}-\vartheta_{n 2}\right)^{\top} \in \mathbb{R}^{2}$ and $\zeta_{o}:=\operatorname{col}\left(\zeta_{1}, \cdots, \zeta_{N}\right) \in \mathbb{R}^{2 N}$. Note that Assumption 1.2 holds for the re-parametrized vectors $\theta_{n}$ as well. A certaintyequivalence adaptive observer for (11) reads as

$$
\begin{aligned}
& \dot{\hat{\zeta}}_{o}=S \hat{\zeta}_{o}+\hat{\boldsymbol{\theta}}(t) u_{a}-\varepsilon \Gamma^{\top}\left[\hat{y}_{a}-y\right] \\
& \hat{y}_{a}=\Gamma \hat{\zeta}_{o}
\end{aligned}
$$

where $\hat{\theta}:=\operatorname{col}\left(\hat{\theta}_{1}, \ldots, \hat{\theta}_{N}\right) \in \mathbb{R}^{2 N}$ collects vectors of estimates $\hat{\theta} \in \mathbb{R}^{2}$, whose update law is to be determined, $\hat{\boldsymbol{\theta}}:=\oplus_{n=1}^{N} \hat{\theta}_{n} \in \mathbb{R}^{2 N \times N}$, and $\varepsilon>0$ is a gain parameter. The design is completed by the certainty-equivalence control

$$
u_{a}=-\varepsilon \hat{\boldsymbol{\theta}}^{\top}(t) \hat{\zeta}_{o}
$$

which yields the closed-loop system in observer coordinates

$$
\dot{\hat{\zeta}}_{o}=\left[S-\varepsilon \hat{\boldsymbol{\theta}}(t) \hat{\boldsymbol{\theta}}^{\top}(t)\right] \hat{\zeta}_{o}-\varepsilon \Gamma^{\top}\left[\hat{y}_{a}-y\right] .
$$

The dynamics of the observation error $\tilde{\zeta}_{o}:=\hat{\zeta}_{o}-\zeta_{o}$ are

$$
\begin{aligned}
& \dot{\tilde{\zeta}}_{o}=F_{\varepsilon} \tilde{\zeta}_{o}+\tilde{\boldsymbol{\theta}}(t) u_{a}+\varepsilon \Gamma^{\top} C(\mu) z \\
& \tilde{y}=\Gamma \tilde{\zeta}_{o}-C(\mu) z
\end{aligned}
$$

where $\tilde{\theta}_{n}:=\hat{\theta}_{n}-\theta_{n}, \tilde{\theta}:=\hat{\theta}-\theta \in \mathbb{R}^{2 N}, \tilde{\boldsymbol{\theta}}:=\oplus_{n=1}^{N} \tilde{\theta}_{n} \in$ $\mathbb{R}^{2 N \times N}$, and $\tilde{y}:=\hat{y}_{a}-y$. Note that $F_{\varepsilon}:=S-\varepsilon \Gamma^{\top} \Gamma \in$ $\mathbb{R}^{2 N \times 2 N}$ is Hurwitz for sufficiently small $\varepsilon>0$ (see Lemma 3.2 in Section III.)

\section{Non-minimal realization of the observer error dynamics}

To design an update law for $\hat{\theta}(t)$, one needs a more convenient parametrization of (15) where the uncertain term $\tilde{\boldsymbol{\theta}}(t)$ is shifted to the output equation. Using a suitable version of the classic Swapping Lemma [20], System (15) is regarded as the parallel interconnection of two systems, with inputoutput maps given respectively by

$$
\tilde{y}_{1}(t)=\Gamma \int_{0}^{t} \mathrm{e}^{F_{\varepsilon}(t-\tau)} \tilde{\boldsymbol{\theta}}(\tau) u_{a}(\tau) \mathrm{d} \tau
$$

and by

$$
\tilde{y}_{2}(t)=\varepsilon \Gamma \int_{0}^{t} \mathrm{e}^{F_{\varepsilon}(t-\tau)} \Gamma^{\top} C(\mu) z(\tau) \mathrm{d} \tau-C(\mu) z(t)
$$

with $\tilde{y}=\tilde{y}_{1}+\tilde{y}_{2}$. The impulse response of (17) admits the LTI realization

$$
\begin{aligned}
& \dot{\xi}_{3}=F_{\varepsilon} \xi_{3}+\varepsilon \Gamma^{\top} C(\mu) z \\
& \tilde{y}_{2}=\Gamma \xi_{3}-C(\mu) z
\end{aligned}
$$

with $\xi_{3} \in \mathbb{R}^{2 N}$, whereas (16) admits the LTV realization

$$
\begin{aligned}
& \dot{\xi}_{1}=E_{\varepsilon}^{\top} \xi_{1}+G u_{a} \\
& \dot{\xi}_{2}=F_{\varepsilon} \xi_{2}+\Xi \dot{\hat{\theta}}(t) \\
& \dot{\Xi}=F_{\varepsilon} \Xi+\boldsymbol{I}\left(u_{a}\right) \\
& \tilde{y}_{1}=\tilde{\theta}^{\top}(t) \xi_{1}-\Gamma \xi_{2}
\end{aligned}
$$

where $\xi_{1} \in \mathbb{R}^{2 N}, \xi_{2} \in \mathbb{R}^{2 N}, \Xi \in \mathbb{R}^{2 N \times 2 N}$ and $E_{\varepsilon}:=$ $S-\varepsilon N G G^{\top} \in \mathbb{R}^{2 N \times 2 N}$. The linear operator $\boldsymbol{I}(\cdot): \mathbb{R}^{N} \rightarrow$ $\mathbb{R}^{2 N \times 2 N}$ is defined as $\boldsymbol{I}\left(u_{a}\right):=\oplus_{n=1}^{N} u_{a_{n}} I_{2}$. The detailed derivation of System (19) is found in Appendix A.

In (19), $\dot{\hat{\theta}}(t)$ is regarded as a known time-varying vector. It will be shown in Lemma 3.6 in Section III that $E_{\varepsilon}^{\top}$ is Hurwitz for all $\varepsilon>0$, therefore System (19) is globally exponentially stable if $\hat{\theta}(\cdot)$ is bounded. While the aggregate state $\operatorname{col}\left(\xi_{2}, \xi_{3}\right)$ is not available for feedback, an open-loop observer of the state $\xi_{1}$ can be easily designed as

$$
\dot{\hat{\xi}}_{1}=E_{\varepsilon}^{\top} \hat{\xi}_{1}+G u_{a} .
$$


Together with (11) and (18), this setup yields the nonlinear closed-loop system

$$
\begin{aligned}
& \dot{\hat{\zeta}}_{o}=\left[S-\varepsilon \hat{\boldsymbol{\theta}} \hat{\boldsymbol{\theta}}^{\top}\right] \hat{\zeta}_{o}-\varepsilon \Gamma^{\top} \tilde{\theta}^{\top} \hat{\xi}_{1}+\varepsilon \Gamma^{\top}\left[\Gamma \xi_{2}-\Gamma \xi_{3}+C(\mu) z\right] \\
& \dot{\hat{\xi}}_{1}=E_{\varepsilon}^{\top} \hat{\xi}_{1}-\varepsilon G \hat{\boldsymbol{\theta}}^{\top} \hat{\zeta}_{o} \\
& \dot{\xi}_{2}=F_{\varepsilon} \xi_{2}+\Xi \varphi \\
& \dot{\Xi}=F_{\varepsilon} \Xi+\boldsymbol{I}\left(-\varepsilon \hat{\boldsymbol{\theta}}^{\top} \hat{\zeta}_{o}\right) \\
& \dot{\xi_{3}}=F_{\varepsilon} \xi_{3}+\varepsilon \Gamma^{\top} C(\mu) z \\
& \dot{z}=A(\mu) z+\varepsilon \Pi(\mu) G \hat{\boldsymbol{\theta}}^{\top} \hat{\zeta}_{o} \\
& \dot{\hat{\theta}}=\varphi \\
& y=\Gamma \hat{\zeta}_{o}-\tilde{\theta}^{\top} \hat{\xi}_{1}+\Gamma \xi_{2}-\Gamma \xi_{3}+C(\mu) z+\tilde{\theta}^{\top} \tilde{\xi}_{1}
\end{aligned}
$$

where $\dot{\hat{\theta}}=\varphi$ is the update law to be determined, and $\tilde{\xi}_{1}:=$ $\hat{\xi}_{1}-\xi_{1}$. Thanks to Lemma 3.6 and the fact that boundedness of $\hat{\theta}(\cdot)$ will be enforced by the selection of $\varphi$, with a minor abuse but no loss of generality, the exponentially decaying term $\tilde{\theta}^{\top} \tilde{\xi}_{1}$ will be dropped from the last equation in (21).

The non-minimal realization (21) of the closed-loop system allows one to recast the original Problem 1 as the following problem of robust output stabilization:

Problem 2: Find an update law $\varphi=\varphi\left(\hat{\zeta}_{o}, \hat{\xi}_{1}, y\right)$ (depending only on the available variables) and a selection for $\varepsilon>0$ such that the trajectories of (21) are bounded and satisfy $\lim _{t \rightarrow \infty} y(t)=0$ for any $\mu \in \mathcal{P}$ and any $\theta \in \Theta^{N}$.

\section{Stability AnAlysis: Baseline ControlleR}

In this section, we provide the stability analysis of the system in closed-loop with the baseline controller (Eq. (21)), and derive sufficient conditions for the update law $\varphi$ to solve Problem 2. The results will serve as a building block for the analysis of the multiple-model algorithm of the next section.

To begin with, we identify desirable properties for $\hat{\theta}(\cdot)$ that will be attained by the update law $\varphi$ designed in Section IV.

Definition 3.1: Let $\hat{\Theta}^{N}$ be the set of $\mathcal{C}^{1}$ signals $\hat{\theta}(\cdot)$ : $\mathbb{R}_{\geq 0} \rightarrow \mathbb{R}^{2 N}$ whose components satisfy

1) $\hat{\theta}_{n}(t) \in \Theta$ for all $t \geq 0$ and $n \in \mathcal{N}$;

2) There exists a constant $\rho>0$ such that $\left\|\dot{\hat{\theta}}_{n}(t)\right\| \leq \varepsilon^{2} \rho$ for all $t \geq 0$ and $n \in \mathcal{N}$

Fix, arbitrarily, $\hat{\theta}(\cdot) \in \hat{\Theta}^{N}$ and regard (21) as the LTV system, with input $u_{1}:=-\tilde{\theta}^{\top} \hat{\xi}_{1} \in \mathbb{R}$ and output $y_{1} \in \mathbb{R}$, defined by

$$
\begin{aligned}
& \dot{\hat{\zeta}}_{o}=\left[S-\varepsilon \hat{\boldsymbol{\theta}}(t) \hat{\boldsymbol{\theta}}^{\top}(t)\right] \hat{\zeta}_{o}-\varepsilon \Gamma^{\top} y_{1}+\varepsilon \Gamma^{\top} u_{1} \\
& \dot{\xi}_{2}=F_{\varepsilon} \xi_{2}+\Xi \dot{\hat{\theta}}(t) \\
& \dot{\Xi}=F_{\varepsilon} \Xi+\boldsymbol{I}\left(-\varepsilon \hat{\boldsymbol{\theta}}^{\top} \hat{\zeta}_{o}\right) \\
& \dot{\xi}_{3}=F_{\varepsilon} \xi_{3}+\varepsilon \Gamma^{\top} C(\mu) z \\
& \dot{z}=A(\mu) z+\varepsilon \Pi(\mu) G \hat{\boldsymbol{\theta}}^{\top}(t) \hat{\zeta}_{o} \\
& y_{1}=-\Gamma \xi_{2}+\Gamma \xi_{3}-C(\mu) z
\end{aligned}
$$

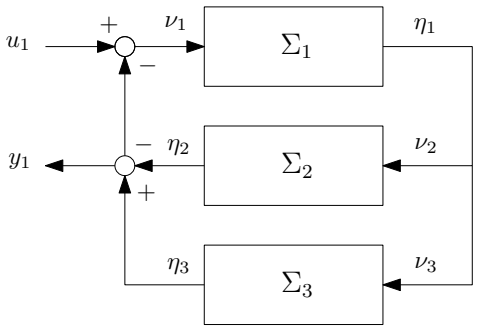

Fig. 3. System (22) as feedback interconnection of (23), (24) and (25).

System (22) is conveniently analyzed as the interconnection of the following linear systems (see Fig. 3) :

$$
\begin{aligned}
& \Sigma_{1}:\left\{\begin{array}{l}
\dot{\hat{\zeta}}_{o}=\left[S-\varepsilon \hat{\boldsymbol{\theta}}(t) \hat{\boldsymbol{\theta}}^{\top}(t)\right] \hat{\zeta}_{o}+\varepsilon \Gamma^{\top} \nu_{1} \\
\eta_{1}=\hat{\boldsymbol{\theta}}^{\top}(t) \hat{\zeta}_{o}
\end{array}\right. \\
& \Sigma_{2}:\left\{\begin{aligned}
\dot{\xi}_{2} & =F_{\varepsilon} \xi_{2}+\Xi \dot{\hat{\theta}}(t) \\
\dot{\Xi} & =F_{\varepsilon} \Xi+\boldsymbol{I}\left(-\varepsilon \nu_{2}\right) \\
\eta_{2} & =\Gamma \xi_{2}
\end{aligned}\right. \\
& \Sigma_{3}:\left\{\begin{aligned}
\dot{\xi}_{3} & =F_{\varepsilon} \xi_{3}+\varepsilon \Gamma^{\top} C(\mu) z \\
\dot{z} & =A(\mu) z+\varepsilon \Pi(\mu) G \nu_{3} \\
\eta_{3} & =\Gamma \xi_{3}-C(\mu) z
\end{aligned}\right.
\end{aligned}
$$

with interconnection structure $\nu_{1}=u_{1}+\eta_{2}-\eta_{3} \in \mathbb{R}, \nu_{2}=$ $\eta_{1} \in \mathbb{R}^{N}, \nu_{3}=\eta_{1} \in \mathbb{R}^{N}$, overall input $u_{1}$ and overall output $y_{1}=\eta_{3}-\eta_{2}$.

Next, we establish properties of Lyapunov function candidates and results for each subsystem in (23)-(25) that are instrumental in the ensuing analysis.

Lemma 3.1: Let $\boldsymbol{I}_{o}:(\theta, \varepsilon) \mapsto \mathbb{R}^{2 N \times 2 N}$ be the positivedefinite diagonal matrix-valued function

$$
\boldsymbol{I}_{o}(\theta, \varepsilon):=\varepsilon \bigoplus_{n=1}^{N} \theta_{n}^{\top} \theta_{n} I_{2}, \quad \varepsilon>0, \theta_{n} \in \Theta
$$

and let $P_{o}:(\theta, \varepsilon) \mapsto \mathbb{R}^{2 N \times 2 N}$ denote the solution of the parameterized family of Lyapunov equations

$$
P_{o}(\theta, \varepsilon)\left[S-\varepsilon \boldsymbol{\theta} \boldsymbol{\theta}^{\top}\right]+\left[S-\varepsilon \boldsymbol{\theta} \boldsymbol{\theta}^{\top}\right]^{\top} P_{o}(\theta, \varepsilon)=-\boldsymbol{I}_{o}(\theta, \varepsilon)
$$

There exist a scalar $\bar{\varepsilon}_{0}>0$ and constants $0<c_{1}<c_{2} \leq c_{3}$ such that $c_{1} I \leq P_{o}(\theta, \varepsilon) \leq c_{2} I$ and $\left\|P_{o}(\theta, \varepsilon)\right\| \leq c_{3}$ for all $(\theta, \varepsilon) \in \Theta^{N} \times\left(0, \bar{\varepsilon}_{0}\right]$.

Lemma 3.2: Let $P_{F}: \varepsilon \mapsto \mathbb{R}^{2 N \times 2 N}$ denote the symmetric solution of the family of Lyapunov equations

$$
P_{F}(\varepsilon) F_{\varepsilon}+F_{\varepsilon}^{\top} P_{F}(\varepsilon)=Q_{F}(\varepsilon)
$$

for some symmetric negative definite matrix-valued function $Q_{F}(\varepsilon)$. There exist scalars $\bar{\varepsilon}_{1} \in \mathbb{R}_{>0}, \lambda \in \mathbb{R}_{>0}, c_{4}>0$, a class- $\mathcal{N}$ function ${ }^{1} c_{5}(\cdot)$ and a symmetric matrix-valued function $Q_{F}(\varepsilon)$ such that for all $\varepsilon \in\left(0, \bar{\varepsilon}_{1}\right]$ :

- $Q_{F}(\varepsilon) \leq-\varepsilon \lambda I_{2 N}$

$$
\text { - } c_{4} I_{2 N} \leq P_{F}(\varepsilon) \leq c_{5}(\varepsilon) I_{2 N} \text {. }
$$

\footnotetext{
${ }^{1}$ A class- $\mathcal{N}$ function $\alpha(\cdot): \mathbb{R}_{+} \rightarrow \mathbb{R}_{+}$is non-negative, continuous and strictly increasing, but not necessarily $\alpha(0)=0$.
} 
Proposition 3.3: There exist scalars $\gamma_{1}^{\star}>0$ and $\varepsilon_{1}^{\star} \in$ $\left(0, \min \left\{\bar{\varepsilon}_{0}, \bar{\varepsilon}_{1}\right\}\right]$ such that $\Sigma_{1}$ is strictly dissipative with respect to the supply rate $q_{1}\left(\nu_{1}, \eta_{1}\right)=\gamma_{1}^{\star 2}\left|\nu_{1}\right|^{2}-\left\|\eta_{1}\right\|^{2}$ for all $\varepsilon \in\left(0, \varepsilon_{1}^{\star}\right)$, with positive definite and decrescent storage function $W_{1}\left(t, \hat{\zeta}_{o}\right)=2 \varepsilon^{-1} \hat{\zeta}_{o}^{\top} P_{o}(\hat{\theta}, \varepsilon) \hat{\zeta}_{o}$.

Proposition 3.4: There exists $\gamma_{2}^{\star}>0$ such that $\Sigma_{2}$ is strictly dissipative with respect to the supply rate $q_{2}\left(\nu_{2}, \eta_{2}\right)=\varepsilon^{2} \gamma_{2}^{\star 2}|| \nu_{2} \|^{2}-\left|\eta_{2}\right|^{2}$ for all $\varepsilon \in\left(0, \varepsilon_{1}^{\star}\right)$, with quadratic and positive definite storage function of the form $W_{2}\left(\xi_{2}, \Xi\right)=a_{1}(\varepsilon) \xi_{2}^{\top} P_{F}(\varepsilon) \xi_{2}+a_{2}(\varepsilon) \operatorname{trace}\left(\Xi^{\top} P_{F}(\varepsilon) \Xi\right)$, for suitable continuous functions $a_{1}(\varepsilon), a_{2}(\varepsilon)>0$.

Proposition 3.5: There exists $\gamma_{3}^{\star}>0$ such that $\Sigma_{3}$ is strictly dissipative with respect to the supply rate $q_{3}\left(\nu_{3}, \eta_{3}\right)=\varepsilon^{2} \gamma_{3}^{\star 2}\left\|\nu_{3}\right\|^{2}-\left|\eta_{3}\right|^{2}$ for all $\varepsilon \in\left(0, \varepsilon_{1}^{\star}\right)$ and all $\mu \in \mathcal{P}$, with a quadratic and positive definite storage function of the form $W_{3}\left(\xi_{3}, z\right)=a_{3}(\varepsilon) \xi_{3}^{\top} P_{F}(\varepsilon) \xi_{3}+a_{4}(\varepsilon) z^{\top} P_{z}(\mu) z$, for suitable continuous functions $a_{3}(\varepsilon), a_{4}(\varepsilon)>0$.

Finally, we establish a Lyapunov function for (20):

Lemma 3.6: Let $P_{E}: \varepsilon \mapsto \mathbb{R}^{2 N \times 2 N}$ be the symmetric solution of the family of Lyapunov equations

$$
P_{E}(\varepsilon) E_{\varepsilon}+E_{\varepsilon}^{\top} P_{E}(\varepsilon)=-\varepsilon N I_{2 N}
$$

Then, there exist a scalar $c_{6}>0$ and a class- $\mathcal{N}$ function $c_{7}(\cdot)$ such that $c_{6} I_{2 N} \leq P_{E}(\varepsilon) \leq c_{7}(\varepsilon) I_{2 N}$ for all $\varepsilon>0 . \triangleleft$ The proofs of Lemmas 3.1, 3.2, 3.6 and Proposition 3.3 are given in the Appendix. Conversely, the proofs of Propositions 3.4 and 3.5 are omitted, as they follow from similar elementary Lyapunov arguments exploiting those lemmas.

For System (22), the following important result, which enables the use of small-gain techniques, is obtained from the analysis of the interconnection structure (23)-(25):

Proposition 3.7: Assume that $\hat{\theta}(\cdot) \in \hat{\Theta}^{N}$. Then, there exists a constant $\varepsilon^{\star} \in \mathbb{R}_{>0}$, which depends only on the compact sets $\mathcal{P}$ and $\Theta$, such that the origin of the unforced System (22) is exponentially stable for any $\varepsilon \in\left(0, \varepsilon^{\star}\right)$. Moreover, the $\mathcal{L}_{2}$-gain between the input $u_{1}$ and the output $y_{1}$ vanishes monotonically as $\varepsilon \rightarrow 0$.

Proof: Fix $\varepsilon \in\left(0, \varepsilon_{1}^{\star}\right)$ and notice that, since the interconnected system is globally Lipschitz uniformly in $t$, solutions exist uniquely for all $t \geq 0$ and all locally essentially bounded inputs $u_{1}(\cdot)$. Applying Propositions 3.3 -3.5 and combining the $\mathcal{L}_{2}$ gains of the single subsystems $\Sigma_{i}, i=1,2,3$, one obtains for all $\tau \in \mathbb{R}_{\geq 0}$

$$
\left\|\eta_{1_{\tau}}\right\|_{\mathcal{L}_{2}} \leq \gamma_{1}^{\star}\left\|u_{1_{\tau}}\right\|_{\mathcal{L}_{2}}+\varepsilon \gamma_{1}^{\star}\left(\gamma_{2}^{\star}+\gamma_{3}^{\star}\right)\left\|\eta_{1_{\tau}}\right\|_{\mathcal{L}_{2}}
$$

where $u_{\tau}(\cdot)$ denotes the truncation of the signal $u(\cdot)$ over $[0, \tau]$. Letting $\varepsilon^{\star}:=\min \left\{\varepsilon_{1}^{\star}, 1 / \gamma_{1}^{\star}\left(\gamma_{2}^{\star}+\gamma_{3}^{\star}\right)\right\}$, it follows that System (22) is a small-gain interconnection (with respect to the $\mathcal{L}_{2}$-norm) for all $\varepsilon \in\left(0, \varepsilon^{\star}\right)$. Application of the smallgain theorem for finite $\mathcal{L}_{2}$-gain systems [26, Theorem 10.8.1 $\&$ Corollary 10.8.2] yields the result, with

$$
\left\|y_{1_{\tau}}\right\|_{\mathcal{L}_{2}} \leq \frac{\varepsilon \gamma_{1}^{\star}\left(\gamma_{2}^{\star}+\gamma_{3}^{\star}\right)}{1-\varepsilon \gamma_{1}^{\star}\left(\gamma_{2}^{\star}+\gamma_{3}^{\star}\right)}\left\|u_{1_{\tau}}\right\|_{\mathcal{L}_{2}}
$$

for all $\varepsilon \in\left(0, \varepsilon^{\star}\right)$ and all $\tau \in \mathbb{R}_{\geq 0}$.
The next result follows from the fact that exponentially stable linear systems are $\mathcal{L}_{2}$-to- $\mathcal{L}_{\infty}$ stable:

Proposition 3.8: Consider the interconnection $\nu_{4}=\eta_{1}$ of System (22) with the LTI system

$$
\Sigma_{4}: \dot{\hat{\xi}}_{1}=E_{\varepsilon}^{\top} \hat{\xi}_{1}-\varepsilon G \nu_{4} .
$$

Under the conditions of Proposition 3.7, the solutions of (22)-(30) belong to $\mathcal{L}_{\infty}$ if $u_{1}(\cdot) \in \mathcal{L}_{2}$.

Proof: From Proposition 3.3, it follows that the storage function $W_{1}\left(t, \hat{\zeta}_{o}\right)$ satisfies the dissipation inequality $\dot{W}_{1} \leq$ $\gamma_{1}^{\star 2}\left|\nu_{1}\right|^{2}-\left\|\eta_{1}\right\|^{2}$. Integrating each side of this inequality along trajectories of (22) and (30) over the interval $[0, \tau]$, and recalling from Proposition 3.4 and Proposition 3.5 that

$$
\left\|\eta_{j_{\tau}}\right\|_{\mathcal{L}_{2}} \leq \varepsilon \gamma_{j}^{\star}\left\|\eta_{1_{\tau}}\right\|_{\mathcal{L}_{2}}, \quad j=2,3,
$$

one obtains

$$
\begin{aligned}
W_{1}\left(\tau, \hat{\zeta}_{o}(\tau)\right)< & W_{1}\left(0, \hat{\zeta}_{o}(0)\right)+\gamma_{1}^{\star 2}\left\|u_{1_{\tau}}\right\|_{\mathcal{L}_{2}}^{2} \\
& -\left(1-\varepsilon^{2} \gamma_{1}^{\star 2}\left(\gamma_{2}^{\star 2}+\gamma_{3}^{\star 2}\right)\right)\left\|\eta_{1_{\tau}}\right\|_{\mathcal{L}_{2}}^{2} .
\end{aligned}
$$

By virtue of Lemma 3.1 and the small-gain condition, this inequality implies that

$$
\left\|\hat{\zeta}_{o}(\tau)\right\| \leq \sqrt{\frac{\varepsilon}{c_{1}}} \max \left\{\sqrt{\frac{2 c_{2}}{\varepsilon}}\left\|\hat{\zeta}_{o}(0)\right\|, \gamma_{1}^{\star}\left\|u_{1}\right\|_{\mathcal{L}_{2}}\right\}
$$

for all $\tau \in \mathbb{R}_{\geq 0}$, hence $\hat{\zeta}_{o}(\cdot) \in \mathcal{L}_{\infty}$. Since $\hat{\theta}(\cdot)$ is bounded by assumption, it follows that $\eta_{1}(\cdot) \in \mathcal{L}_{\infty}$. Exponential stability of Systems (24), (25) and (30) implies that all remaining state trajectories belong to $\mathcal{L}_{\infty}$ as well.

Combining Proposition 3.7 and Proposition 3.8, one obtains the main result of this section:

Theorem 3.9: Let Assumptions 1.1 and 1.2 hold. If there exists an update law $\varphi=\varphi\left(\hat{\zeta}_{o}, \hat{\xi}_{1}, y\right)$ such that:

(i.) the forward solution $\hat{\theta}(\cdot)$ of $\dot{\hat{\theta}}=\varphi\left(\hat{\zeta}_{o}, \hat{\xi}_{1}, y\right)$ belongs to $\hat{\Theta}^{N}$ for all initial conditions $\hat{\theta}(0) \in \operatorname{int} \Theta^{N}$;

(ii.) the signal $\eta_{4}(\cdot):=\hat{\xi}_{1}^{\top}(\cdot) \tilde{\theta}(\cdot)$ belongs to $\mathcal{L}_{2}$.

Then there exists a constant $\underline{\varepsilon}$ such that the problem of output stabilization for System (21) is solved for all $\varepsilon \in(0, \underline{\varepsilon})$. $\triangleleft$

Proof: Notice that Assumptions 1.1-1.2 and (i.)-(ii.) imply that the requirements of Propositions 3.7 and 3.8 are satisfied. Setting $u_{1}(\cdot)=-\eta_{4}(\cdot)$ in System (22), Condition (ii.) implies that $\hat{\xi}_{1}(\cdot) \in \mathcal{L}_{\infty}$ and $\dot{\hat{\xi}}_{1}(\cdot) \in \mathcal{L}_{\infty}$ for all $\varepsilon \in(0, \underline{\varepsilon})$. Since $\hat{\theta}(\cdot) \in \hat{\Theta}^{N}$ implies that $\hat{\theta}(\cdot) \in \mathcal{L}_{\infty}$ and $\dot{\hat{\theta}}(\cdot) \in \mathcal{L}_{\infty}$, it follows that $\eta_{4}(\cdot) \in \mathcal{L}_{2} \cap \mathcal{L}_{\infty}$ and $\dot{\eta}_{4}(\cdot) \in \mathcal{L}_{\infty}$. By a corollary of Barbălat's Lemma [20, Lemma 3.2.5], it follows that $\lim _{t \rightarrow \infty} \eta_{4}(t)=0$. Since System (22) is exponentially stable for all $\varepsilon \in(0, \underline{\varepsilon})$, it follows that $\hat{\zeta}_{o}(t)$, $\xi_{2}(t), \xi_{3}(t), \Xi(t)$ and $z(t)$ vanish asymptotically, hence $\lim _{t \rightarrow \infty} y(t)=0$.

\section{Multiple-Model Switching CONTROller}

The results of Section III have shifted the burden of the whole control strategy of Section II to the problem of finding a suitable update law with the required characteristics listed 


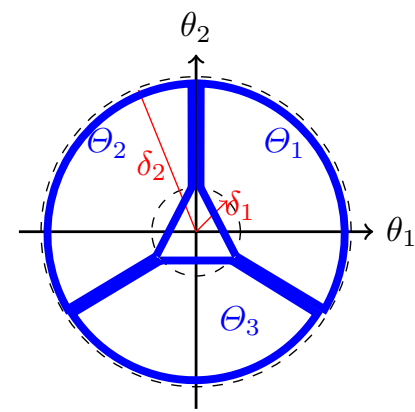

Fig. 4. Finite covering of $\Theta$ using three convex sets, $\Theta_{i} i=1,2,3$.

in the statement of Theorem 3.9. While boundedness of $\dot{\hat{\theta}}(\cdot)$ can be enforced via normalization, ensuring that $\hat{\theta}(t) \in \Theta^{N}$ for all $t \geq 0$ is hindered by the fact that $\Theta$ is not convex, hence standard projection techniques do not apply. Following the seminal work [21] and later enhancements [22]-[25], the parameter set $\Theta$ defined in (7) is extended to a finite covering of convex sets. In particular ${ }^{2}$, we let $\bar{\Theta}:=\bigcup_{k=1}^{3} \Theta_{k}$ as shown in Figure 4, and replace $\Theta$ with $\bar{\Theta}$ and $\Theta^{N}$ with $\bar{\Theta}^{N}$. Denoting the index set by $\mathcal{Q}:=\left\{1,2, \cdots, 3^{N}\right\}$, different parameter estimate vectors $\hat{\theta}^{i} \in \mathbb{R}^{2 N}, i \in \mathcal{Q}$ are introduced, each governed by its own update law. Since one must consider all possible combinations of estimates $\hat{\theta}_{n}^{i}$ for each frequency $\omega_{n}$ belonging to each one of the three sets $\Theta_{k}, k \in\{1,2,3\}, \mathcal{Q}$ is necessarily the power set. The map

$$
\mathcal{R}(i, n): \mathcal{Q} \times \mathcal{N} \mapsto\{1,2,3\}
$$

establishes a one-to-one relation among estimators, distinct excitation frequencies and corresponding parameter subsets. This map is specified in such a way that $\cup_{i} \mathcal{R}(i, \cdot)$ covers all $3^{N}$ combinations of the parameter sets, that is $\cup_{n} \mathcal{R}(i, n) \neq$ $\cup_{n} \mathcal{R}(j, n)$, for any $i, j \in \mathcal{Q}, i \neq j$. The ensuing partition of the parameter space leads one to replace the original adaptive controller with a switching controller based on a multiple model estimator, where $3^{N}$ estimators are continuously run in parallel, whereas at a given time only one adaptive controller (corresponding to a specific estimator) is connected to the plant input. The selection of the active controller is demanded to the switching logic with hysteresis of [22], [25]. The structure of the overall controller, shown in Figure 5, comprises the following modules:

1) Multiple-model Estimator and Switching Controller: The observer-based adaptive controller (12)-(13) is replaced by controllers of the form

$$
\begin{aligned}
& \dot{\hat{\zeta}}_{o}^{i}=S \hat{\zeta}_{o}^{i}+\hat{\boldsymbol{\theta}}^{i} u_{a}-\varepsilon \Gamma^{\top}\left[\hat{y}_{a}^{i}-y\right], \quad \hat{\zeta}_{o}^{i} \in \mathbb{R}^{2 N} \\
& \hat{y}_{a}^{i}=\Gamma \hat{\zeta}_{o}^{i} \\
& u_{a}^{i}=-\varepsilon \hat{\boldsymbol{\theta}}^{i \top} \hat{\zeta}_{o}^{i}, \quad i \in \mathcal{Q} .
\end{aligned}
$$

The control signal applied to the plant at any $t \geq 0$ is $u_{a}(t)=$ $u_{a}^{\sigma(t)}(t)$, being $\sigma: \mathbb{R}_{\geq 0} \rightarrow \mathcal{Q}$ a piecewise-constant switching signal determined by the switching mechanism.

\footnotetext{
${ }^{2}$ We consider one of many solutions to convexification of $\Theta$ with a minimum number of subsets. One can arbitrarily modify the subsets in Figure 4, as long as they remain convex and with non-overlapping interior.
}

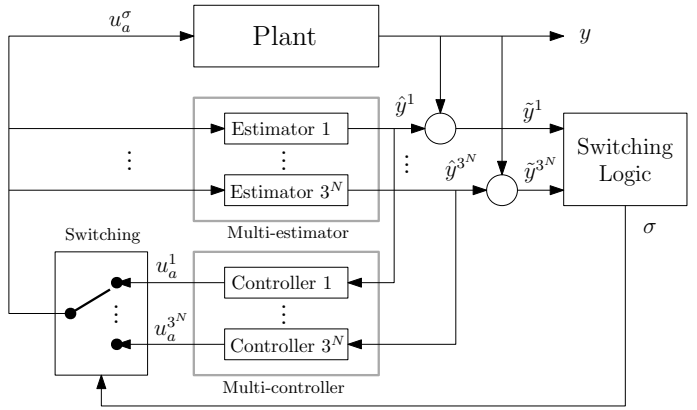

Fig. 5. Block diagram of the multiple-model controller.

2) Multiple-model Update Law: For each vector $\hat{\theta}^{i}$, the parameter estimate $\hat{\theta}_{n}^{i}$, associated to frequency $\omega_{n}$, is constrained to evolve on one of the convex sets $\Theta_{k}, k=\mathcal{R}(i, n)$. The corresponding update law is given by

$$
\begin{aligned}
& \dot{\hat{\theta}}_{n}^{i}=\operatorname{Proj}_{k}\left(\hat{\theta}_{n}^{i}, \varphi_{n}^{i}\left(\hat{\xi}_{1}, \tilde{y}^{i}\right)\right), \quad \hat{\theta}_{n}^{i}(0) \in \operatorname{int} \Theta_{k} \\
& \dot{\hat{\xi}}_{1}=E_{\varepsilon}^{\top} \hat{\xi}_{1}+G u_{a}, \quad \hat{\xi}_{1}(0) \in \mathbb{R}^{2 N} \\
& \tilde{y}^{i}=\hat{y}_{a}^{i}-y, \quad i \in \mathcal{Q}, \quad n \in \mathcal{N}
\end{aligned}
$$

where $k=\mathcal{R}(i, n)$ and $\operatorname{Proj}_{k}(\cdot)$ is the standard projection on $\Theta_{k}$ [20]. Note that all update laws share the state of a single observer for the state $\xi_{1}$ of (19). The unconstrained update law $\varphi^{i}:\left(\hat{\xi}_{1}, \tilde{y}^{i}\right) \mapsto \mathbb{R}^{2 N}$ for each estimator is selected as the normalized gradient law

$$
\varphi_{n}^{i}\left(\hat{\xi}_{1}, \tilde{y}^{i}\right)=-\frac{\rho \varepsilon^{2}}{N} \frac{\hat{\xi}_{1 n} \tilde{y}^{i}}{m_{i}^{2}} \in \mathbb{R}^{2}, \quad n \in \mathcal{N}, i \in \mathcal{Q}
$$

yielding $\varphi^{i}\left(\hat{\xi}_{1}, \tilde{y}^{i}\right)=\operatorname{col}\left(\varphi_{1}^{i}\left(\hat{\xi}_{11}, \tilde{y}^{i}\right), \ldots, \varphi_{N}^{i}\left(\hat{\xi}_{1 N}, \tilde{y}^{i}\right)\right)$. The normalization signal $m_{i}^{2}:=1+\left\|\hat{\xi}_{1}\right\|^{2}+\left|\tilde{y}^{i}\right|^{2}$ ensures that $\left\|\varphi^{i}\right\|<\rho \varepsilon^{2}$ for all $i \in \mathcal{Q}$.

3) Hysteresis Switching Logic: As in [23], the following functions are defined as the performance criteria employed in the switching logic for all estimator/controller pairs:

$$
J^{i}(t)=\beta \int_{0}^{t} \tilde{y}^{i}(\tau)^{2} d \tau, \quad i \in \mathcal{Q}
$$

where $\beta>0$ is a parameter to be selected. The hysteresis switching logic of [25] is adopted for the controller selection.

To specify the mechanism of hysteresis switching, it is necessary to select the hysteresis constant $h \in \mathbb{R}_{>0}$. First, set $\sigma(0)=\arg \min _{i \in \mathcal{Q}}\left\{J^{i}(0)\right\}$. Suppose that at certain time $t_{m}>0$, the value of $\sigma\left(t_{m}\right)$ just switches to some $i_{0} \in \mathcal{Q}$. Then $\sigma(t)$ is kept constant until the time $t_{m+1}>t_{m}$ such that $h+\min _{i \in \mathcal{Q}}\left\{J^{i}\left(t_{m+1}\right)\right\}<J^{i_{0}}\left(t_{m+1}\right)$; at that point, set $\sigma\left(t_{m+1}\right)=\arg \min _{i \in \mathcal{Q}}\left\{J^{i}\left(t_{m+1}\right)\right\}$. Repeating the above steps, one generates a piecewise-constant signal $\sigma(t)$ which is continuous from the right everywhere. When the indicated arg min is not unique, a particular value for $\sigma$ among those achieving the minimum can be selected arbitrarily.

Let the time sequence $\left\{T_{m}\right\}_{m=1}^{M}$, denote the times at which the switching takes place; note that $T_{0}=0, \sigma\left(T_{0}\right)=$ 
$\operatorname{argmin}\left\{J^{i}(0)\right\}$ and that $M \leq \infty$. The switching logic is formally stated as

$$
\sigma\left(T_{m}\right):=\arg \min _{i \in \mathcal{Q}, i \neq k}\left\{J_{h}^{i}\left(T_{m}\right)\right\}
$$

where $J_{h}^{i}\left(T_{m}\right):=J^{i}\left(T_{m}\right)+h$ and $k=\sigma\left(T_{m-1}\right)$ is the index identifying the previously activating controller. According to the hysteresis switching mechanism, a switching occurs at $t=T_{m}$ only if the condition

$$
J^{k}\left(T_{m}\right)>\min _{i \in \mathcal{Q}, i \neq k}\left\{J_{h}^{i}\left(T_{m}\right)\right\}
$$

is satisfied. Note that $\sigma(t)=\sigma\left(T_{m}\right)$ in any time interval $\left[T_{m}, T_{m+1}\right)$. The proposed switching scheme thus consists of continuously monitoring the performance index $J_{h}^{i}(t)$, $i \in \mathcal{Q}$. Using the arguments in [28], it can be shown that on each interval $[0, T]$ there are only finite number of switches, therefore the infinitely fast switching (chattering) phenomenon is avoided. At each $t$, the controller connected to the plant is selected as $u_{a}(t)=u_{a}^{\sigma(t)}(t)$. In the sequel, we drop the dependence of $\sigma(t)$ on $t$ when letting $\sigma$ represent the active controller.

The multiple-model switching controller (32)-(34) yields the new closed-loop system

$$
\begin{aligned}
& \dot{\hat{\zeta}}_{o}^{i}=S \hat{\zeta}_{o}^{i}-\varepsilon \hat{\boldsymbol{\theta}}^{i} \hat{\boldsymbol{\theta}}^{\sigma \top} \hat{\zeta}_{o}^{\sigma}-\varepsilon \Gamma^{\top} \tilde{\theta}^{i \top} \hat{\xi}_{1}+\varepsilon \Gamma^{\top}\left[\Gamma \xi_{2}-\Gamma \xi_{3}+C(\mu) z\right] \\
& \dot{\hat{\xi}}_{1}=E_{\varepsilon}^{\top} \hat{\xi}_{1}-\varepsilon G \hat{\boldsymbol{\theta}}^{\sigma \top} \hat{\zeta}_{o}^{\sigma} \\
& \dot{\xi}_{2}=F_{\varepsilon} \xi_{2}+\Xi \dot{\hat{\theta}}^{\sigma} \\
& \dot{\Xi}=F_{\varepsilon} \Xi+\boldsymbol{I}\left(-\varepsilon \hat{\boldsymbol{\theta}}^{\sigma \top} \hat{\zeta}_{o}^{\sigma}\right) \\
& \dot{\xi}_{3}=F_{\varepsilon} \xi_{3}+\varepsilon \Gamma^{\top} C(\mu) z \\
& \dot{z}=A(\mu) z+\varepsilon \Pi(\mu) G \hat{\boldsymbol{\theta}}^{\sigma \top} \hat{\zeta}_{o}^{\sigma} \\
& \dot{\hat{\theta}_{n}^{i}}=\operatorname{Proj}_{k}\left(\hat{\theta}_{n}^{i}, \varphi_{n}^{i}\left(\hat{\xi}_{1 n}, \tilde{y}^{i}\right)\right) \\
& y=\Gamma \hat{\zeta}_{o}^{i}-\tilde{\theta}^{i \top} \hat{\xi}_{1}+\Gamma \xi_{2}-\Gamma \xi_{3}+C(\mu) z \\
& \tilde{y}^{i}=\Gamma \hat{\zeta}_{o}^{i}-y
\end{aligned}
$$

where $i \in \mathcal{Q}, n \in \mathcal{N}$ and $k=\mathcal{R}(i, n) \in\{1,2,3\}$.

\section{Stability Analysis: Switching Controller}

In this section, we complete the stability and convergence analysis of the multiple-model extension of the baseline controller. Referring to the results presented in Section III, the outstanding issues are to show that Theorem 3.9 holds for the multiple-model estimator-based switching controller, and that the properties (i) and (ii) listed therein are verified.

We begin by noticing that if there exists a time $T_{s}>0$ after which the switchings stop, then for all $t \geq T_{s}$ the active subsystem of the multiple-model controller of Section IV is equivalent to the baseline controller of Section II. Specifically, after the switching stops, the active states (i.e. $\hat{\zeta}_{o}^{\sigma}, \hat{\xi}_{1}$, $\xi_{2}, \Xi, \xi_{3}, z$ and $\hat{\theta}^{\sigma}$ ) of the multiple-model estimator-based system (37) admits the same dynamics as the System (21). Consequently, Theorem 3.9 must hold for System (37), as the inactive subsystems (with states $\hat{\zeta}_{o}^{i}, \hat{\theta}^{i}$, for $i \in \mathcal{Q} \backslash\{\sigma\}$ ) are disconnected from the rest of the system. Therefore, finite termination of the switching sequence $\left\{T_{m}\right\}_{m=1}^{M}$ is key for the analysis. A series of intermediate results, presented next, will be instrumental in showing that $T_{M}<\infty$.

Consider the new closed-loop system (37), where the feedback interconnection structure in Fig. 3 is preserved with input $u_{1}=-\tilde{\theta}^{\sigma} \top \hat{\xi}_{1} \in \mathbb{R}$. First, it is readily seen that in any time interval $\left[T_{m-1}, T_{m}\right)$, the analogous of Proposition 3.3 holds for the new subsystem $\Sigma_{1}$ :

$$
\Sigma_{1}:\left\{\begin{array}{l}
\dot{\hat{\zeta}}_{o}^{\sigma}=\left[S-\varepsilon \hat{\boldsymbol{\theta}}^{\sigma} \hat{\boldsymbol{\theta}}^{\sigma \top}\right] \hat{\zeta}_{o}^{\sigma}+\varepsilon \Gamma^{\top} \nu_{1} \\
\eta_{1}=\hat{\boldsymbol{\theta}}^{\sigma} \hat{\zeta}_{o}^{\sigma}
\end{array}\right.
$$

with positive definite and decrescent storage function $W_{1}^{\prime}\left(t, \hat{\zeta}_{o}^{\sigma}\right)=2 \varepsilon^{-1} \hat{\zeta}_{o}^{\sigma \top} P_{o}\left(\hat{\theta}^{\sigma}(t), \varepsilon\right) \hat{\zeta}_{o}^{\sigma}$. Moreover, as the crucial property $\left\|\hat{\theta}^{\sigma(t)}(t)\right\| \leq \rho \varepsilon^{2}$ is preserved, it is not surprising that within each interval $\left[T_{m-1}, T_{m}\right)$ the analogous of Proposition 3.4 holds for the new subsystem $\Sigma_{2}$ defined as

$$
\Sigma_{2}:\left\{\begin{aligned}
\dot{\xi}_{2} & =F_{\varepsilon} \xi_{2}+\Xi \dot{\hat{\theta}}^{\sigma} \\
\dot{\Xi} & =F_{\varepsilon} \Xi+\boldsymbol{I}\left(-\varepsilon \nu_{2}\right) \\
\eta_{2} & =\Gamma \xi_{2}
\end{aligned}\right.
$$

Finally, using the appropriate modifications of Propositions 3.3-3.4 and following the same lines of the proof of Proposition 3.7 , one can show that the finite $\mathcal{L}_{2}$-gain properties established in Proposition 3.7 are preserved. Towards this end, for any time interval $[0, \tau]$, let $[0, \tau]=$ $\bigcup_{m=1}^{\bar{m}}\left[T_{m-1}, T_{m}\right) \bigcup\left[T_{\bar{m}}, \tau\right]$ where $0 \leq T_{\bar{m}} \leq \tau<T_{\bar{m}+1}$. From elementary properties of Lebesgue integral [29, Theorem 4, Sec.29.2], it follows that

$$
\begin{aligned}
\left\|y_{1_{\tau}}\right\|_{\mathcal{L}_{2}}^{2} & =\sum_{m=1}^{\bar{m}} \int_{T_{m-1}}^{T_{m}}\left|y_{1}(s)\right|^{2} d s+\int_{T_{\bar{m}}}^{\tau}\left|y_{1}(s)\right|^{2} d s \\
& \leq \hat{\varepsilon}^{2}(\varepsilon) \sum_{m=1}^{\bar{m}} \int_{T_{m-1}}^{T_{m}}\left|u_{1}(s)\right|^{2} d s+\hat{\varepsilon}^{2}(\varepsilon) \int_{T_{\bar{m}}}^{\tau}\left|u_{1}(s)\right|^{2} d s \\
& =\hat{\varepsilon}^{2}(\varepsilon)\left\|u_{1_{\tau}}\right\|_{\mathcal{L}_{2}}^{2}
\end{aligned}
$$

with $\hat{\varepsilon}(\varepsilon):=\frac{\varepsilon \gamma_{1}^{\star}\left(\gamma_{2}^{\star}+\gamma_{3}^{\star}\right)}{1-\varepsilon \gamma_{1}^{\star}\left(\gamma_{2}^{\star}+\gamma_{3}^{\star}\right)}$, from which one concludes that

$$
\left\|y_{1_{\tau}}\right\|_{\mathcal{L}_{2}} \leq \hat{\varepsilon}(\varepsilon)\left\|u_{1_{\tau}}\right\|_{\mathcal{L}_{2}}
$$

for all $\varepsilon \in\left(0, \varepsilon^{\star}\right)$ and all $\tau \in \mathbb{R}_{\geq 0}$, which is consistent with Proposition 3.7. These results lead to the claim that the switching mechanism stops in finite time:

Proposition 5.1: For the hysteresis switching mechanism in (36), there exist constant $\underline{\varepsilon}_{0}$ and a finite instant $T_{s}>0$ such that for all $\varepsilon \in\left(0, \underline{\varepsilon}_{0}\right]$

$$
\sigma(t)=\sigma\left(T_{s}\right) \quad \forall t \in\left[T_{s},+\infty\right)
$$

that is, switchings stop at most in $T_{s}$ units of time.

The proof of Proposition 5.1 is given in the Appendix.

Next, we show that Proposition 3.8 (finite $\mathcal{L}_{2}$-to- $\mathcal{L}_{\infty}$ gain) holds in the interval $\left[T_{s},+\infty\right)$ for the active subsystems $\Sigma_{i}, i=1, \ldots, 4$, of the new closed-loop system (37). From the revised Proposition 3.3, it follows that the storage function $W_{1}^{\prime}\left(t, \hat{\zeta}_{o}^{\sigma}\right)$ satisfies the dissipation inequality $\dot{W}_{1}^{\prime} \leq$ $\gamma_{1}^{\star^{2}}\left|\nu_{1}\right|^{2}-\left\|\eta_{1}\right\|^{2}$ as well. Integrating each side of this 
inequality along trajectories of (37) over the interval $\left[T_{s}, t\right)$, and recalling from Proposition 3.4 and Proposition 3.5 that

$$
\int_{T_{s}}^{t}\left|\eta_{j}(s)\right|^{2} d s \leq \varepsilon^{2} \gamma_{j}^{\star 2} \int_{T_{s}}^{t}\left\|\eta_{1}(s)\right\|^{2} d s \quad j=2,3,
$$

one obtains

$$
\begin{aligned}
W_{1}^{\prime}\left(t, \hat{\zeta}_{o}^{\sigma}(t)\right)< & W_{1}^{\prime}\left(T_{s}, \hat{\zeta}_{o}^{\sigma}\left(T_{s}\right)\right)+\gamma_{1}^{\star 2} \int_{T_{s}}^{t}\left|u_{1}(s)\right|^{2} d s \\
& -\left(1-\varepsilon^{2} \gamma_{1}^{\star 2}\left(\gamma_{2}^{\star 2}+\gamma_{3}^{\star 2}\right)\right) \int_{T_{s}}^{t}\left\|\eta_{1}(s)\right\|^{2} d s .
\end{aligned}
$$

This implies, by virtue of Lemma 3.1 and the small-gain interconnection, that

$$
\left\|\hat{\zeta}_{o}^{\sigma}(t)\right\| \leq \sqrt{\frac{\varepsilon}{c_{1}}} \max \left\{\sqrt{\frac{2 c_{2}}{\varepsilon}}\left\|\hat{\zeta}_{o}^{\sigma}\left(T_{s}\right)\right\|, \gamma_{1}^{\star}\left(\int_{T_{s}}^{t}\left|u_{1}(s)\right|^{2} d s\right)^{\frac{1}{2}}\right\}
$$

for all $t \in\left[T_{s},+\infty\right)$. Hence, $\hat{\zeta}_{o}^{\sigma}(\cdot) \in \mathcal{L}_{\infty}$. Since $\hat{\theta}^{\sigma}(\cdot)$ is bounded by construction of the update laws, it follows that $\eta_{1}(\cdot) \in \mathcal{L}_{\infty}$. Exponential stability of new Systems (39), (25) and (30) implies that state trajectories of system $\Sigma_{2}, \Sigma_{3}$ and $\Sigma_{4}$ belong to $\mathcal{L}_{\infty}$ as well. Given the revised Proposition 3.8, it follows that Theorem. 3.9 holds for the multiple-model estimator-based system (37) for all $t \geq T_{s}$.

The remaining issue is to show that properties (i.) and (ii.) of Theorem 3.9 are satisfied. For the adaptation law defined in (33), the following result is readily obtained:

Proposition 5.2: For all $i \in \mathcal{Q}$ and $n \in \mathcal{N}$, the forward solutions $\hat{\theta}^{i}(\cdot)$ of (33)-(34) are defined over $[0, \infty)$ and satisfy $\hat{\theta}_{n}^{i}(t) \in \hat{\Theta}_{k}$ and $\left\|\dot{\hat{\theta}}^{i}(t)\right\| \leq \rho \varepsilon^{2}$, for all $t \geq 0$ and all initial conditions $\hat{\theta}_{n}^{i}(0) \in \operatorname{int} \Theta_{k}, k \in\{1,2,3\}$.

Hence, condition (i.) of Thm. 3.9 is verified for $\hat{\theta}^{\sigma}(t)$, in particular for all $t \in\left[T_{s},+\infty\right)$. Checking condition (ii.) requires significantly more work. First, one more intermediate result is needed:

Lemma 5.3: The normalization signal $m_{i}(\cdot)$ in the update law (34) belongs to $\mathcal{L}_{\infty}$ for all $i \in \mathcal{Q}$.

The proof, found in the Appendix, is established using classic arguments based on exponentially weighted norms [20].

Finally, we are in the position to show that the crucial requirement (ii.) of Theorem 3.9, namely that $\eta_{4}(t) \in \mathcal{L}_{2}$, holds for the multiple-model estimator-based switching controller. Consider the storage function $V^{\kappa}\left(\tilde{\theta}^{\kappa}\right)=\frac{1}{2}\left\|\tilde{\theta}^{\kappa}\right\|^{2}$ where $\tilde{\theta}^{\kappa}:=\hat{\theta}^{\kappa}-\theta$, and $\kappa$ labels the estimator whose parameters $\hat{\theta}_{n}^{\kappa}$ are all constrained on the regions that contain the true parameter vector $\theta_{n}$, i.e., such that $\theta_{n} \in \Theta_{\mathcal{R}(\kappa, n)}$, for all $n \in \mathcal{N}$. Evaluating the derivative of $V^{\kappa}\left(\tilde{\theta}^{\kappa}\right)$ along the trajectories of the parameter estimates governed by (33) and (34) yields

$$
\begin{aligned}
\dot{V}^{\kappa} & =\operatorname{Proj}_{k}\left(\hat{\theta}_{n}^{\kappa}, \varphi_{n}^{\kappa}\left(\hat{\xi}_{1}, \tilde{y}^{\kappa}\right)\right) \\
& =-\rho \varepsilon^{2} \tilde{\theta}^{\kappa \top} \frac{\hat{\xi}_{1} \tilde{y}^{\kappa}}{m_{\kappa}^{2}}-\tilde{\theta}^{\kappa \top} \mathcal{I}_{\kappa}\left(\hat{\theta}^{\kappa}, \varphi^{\kappa}\right) \varphi^{\kappa}\left(\hat{\xi}_{1}, \tilde{y}^{\kappa}\right)
\end{aligned}
$$

with obvious definition of $\mathcal{I}_{\kappa}(\cdot)$. Since $\theta_{n} \in \Theta_{\mathcal{R}(\kappa, n)}$, the term $\tilde{\theta}^{\kappa \top} \mathcal{I}_{\kappa}\left(\hat{\theta}^{\kappa}, \varphi^{\kappa}\right) \varphi^{\kappa}\left(\hat{\xi}_{1}, \tilde{y}^{\kappa}\right)$ is non-negative, hence

$$
\dot{V}^{\kappa} \leq-\rho \varepsilon^{2} \tilde{\theta}^{\kappa \top} \frac{\hat{\xi}_{1} \tilde{y}^{\kappa}}{m_{\kappa}^{2}}
$$

Substituting the relation $\tilde{y}^{\kappa}=\hat{\xi}_{1}^{\top} \tilde{\theta}^{\kappa}+y_{1}$ and integrating both sides of (41) from $T_{s}$ to $t$, one obtains

$$
\begin{array}{r}
V^{\kappa}(t)-V^{\kappa}\left(T_{s}\right) \leq-\frac{\rho \varepsilon^{2}}{2\left\|m_{\kappa}\right\|_{\infty}^{2}} \int_{T_{s}}^{t}\left|\hat{\xi}_{1}(\tau)^{\top} \tilde{\theta}^{\kappa}(\tau)\right|^{2} d \tau \\
+\frac{\rho \varepsilon^{2}}{2} \int_{T_{s}}^{t}\left|y_{1}(\tau)\right|^{2} d \tau
\end{array}
$$

where we have taken advantage of boundedness of $m_{\kappa}(\cdot)$ established in Lemma 5.3. As before, we label with $\sigma$ the active controller. Two scenarios must be considered separately: In the first one, if $\sigma\left(T_{s}\right)=\kappa$, then $\eta_{4}(t)=u_{1}(t)=\hat{\xi}_{1}^{\top}(t) \tilde{\theta}^{\kappa}(t)$. Applying the small-gain condition (40) to (42) one obtains:

$$
\frac{\rho \varepsilon^{2}}{2}\left(\frac{1}{\left\|m_{\kappa}\right\|_{\infty}^{2}}-\hat{\varepsilon}^{2}(\varepsilon)\right) \int_{T_{s}}^{t}\left|\eta_{4}(\tau)\right|^{2} d \tau \leq V^{\kappa}\left(T_{s}\right)
$$

for all $\varepsilon \in\left(0, \varepsilon^{\star}\right)$, where $\hat{\varepsilon}(\varepsilon):=\frac{\varepsilon \gamma_{1}^{\star}\left(\gamma_{2}^{\star}+\gamma_{3}^{\star}\right)}{1-\varepsilon \gamma_{1}^{\star}\left(\gamma_{2}^{\star}+\gamma_{3}^{\star}\right)}$. By Proposition $3.7, \hat{\varepsilon}^{2}(\varepsilon)$ vanishes monotonically as $\varepsilon \rightarrow 0$. Thus, there exists a constant $\underline{\varepsilon}_{1}$ such that, for all $\varepsilon \in\left(0, \underline{\varepsilon}_{1}\right)$, $1 /\left\|m_{\kappa}\right\|_{\infty}^{2}-\hat{\varepsilon}^{2}(\varepsilon)>0$. In this case, it follows that

$$
\int_{T_{s}}^{t}\left|\eta_{4}(\tau)\right|^{2} d \tau \leq \frac{2\left\|m_{\kappa}\right\|_{\infty}^{2}}{\rho \varepsilon^{2}\left(1-\left\|m_{\kappa}\right\|_{\infty}^{2} \hat{\varepsilon}^{2}\right)} V^{\kappa}\left(T_{s}\right)<\infty
$$

for all $t \geq T_{s}$ and for all $\varepsilon \in\left(0, \min \left\{\varepsilon^{\star}, \underline{\varepsilon}_{0}, \underline{\varepsilon}_{1}\right\}\right)$.

In the second situation where $\sigma\left(T_{s}\right) \neq \kappa$, hence $\eta_{4}(t)=$ $\hat{\xi}_{1}^{\top}(t) \tilde{\theta}^{\sigma}$, let us define $\bar{\eta}_{4}(t):=\hat{\xi}_{1}^{\top}(t) \tilde{\theta}^{\kappa}(t)$. Referring to (35), the error variable $\bar{e}(t):=J^{\kappa}(t)-J^{\sigma}(t)$ satisfies

$$
\bar{e}(t)=\bar{e}\left(T_{s}\right)+\beta \int_{T_{s}}^{t}\left(\tilde{y}^{\kappa}(\tau)\right)^{2}-\left(\tilde{y}^{\sigma}(\tau)\right)^{2} d \tau \quad \forall t \geq T_{s} .
$$

According to the hysteresis switching logic (36), it follows that $\bar{e}\left(T_{s}\right) \geq 0$ and $\bar{e}(t) \geq-h$ for all $t>T_{s}$. Consequently,

$$
\int_{T_{s}}^{t}\left(\tilde{y}^{k}(\tau)\right)^{2}-\left(\tilde{y}^{j}(\tau)\right)^{2} d \tau \geq-\frac{1}{\beta}\left(h+\bar{e}\left(T_{s}\right)\right):=-c_{\bar{e}}
$$

with a positive constant $c_{\bar{e}}$. Applying the inequalities

$$
\begin{gathered}
\left|\tilde{y}^{\kappa}\right|^{2}=\left|\bar{\eta}_{4}\right|^{2}+\left|y_{1}\right|^{2}+2\left|\bar{\eta}_{4}\right|\left|y_{1}\right| \leq 2\left|\bar{\eta}_{4}\right|^{2}+2\left|y_{1}\right|^{2} \\
\left|\tilde{y}^{\sigma}\right|^{2}=\left|\eta_{4}\right|^{2}+\left|y_{1}\right|^{2}+2\left|\eta_{4}\right|\left|y_{1}\right| \geq \frac{1}{2}\left|\eta_{4}\right|^{2}-\left|y_{1}\right|^{2}
\end{gathered}
$$

to (44), yields

$$
\begin{aligned}
-c_{\bar{e}} \leq \int_{T_{s}}^{t}\left[\left(\tilde{y}^{\kappa}(\tau)\right)^{2}-\left(\tilde{y}^{j}(\tau)\right)^{2}\right] d \tau \\
\quad \leq \int_{T_{s}}^{t} 2\left|\bar{\eta}_{4}(\tau)\right|^{2}-\frac{1}{2}\left|\eta_{4}(\tau)\right|^{2}+3\left|y_{1}(\tau)\right|^{2} d \tau
\end{aligned}
$$

from which it follows that

$$
-\int_{T_{s}}^{t}\left|\bar{\eta}_{4}(\tau)\right|^{2} d \tau \leq-\frac{1}{4} \int_{T_{s}}^{t}\left|\eta_{4}(\tau)\right|^{2} d \tau+\frac{3}{2} \int_{T_{s}}^{t}\left|y_{1}(\tau)\right|^{2} d \tau+\frac{c_{\bar{e}}}{2} .
$$


Substituting the above expression into (42) yields the dissipation inequality

$$
\begin{aligned}
& V^{\kappa}(t)-V^{\kappa}\left(T_{s}\right) \leq \frac{\rho \varepsilon^{2}}{2} \int_{T_{s}}^{t}\left|y_{1}(\tau)\right|^{2} d \tau \\
& +\frac{\rho \varepsilon^{2}}{2\left\|m_{\kappa}\right\|_{\infty}^{2}}\left[-\frac{1}{4} \int_{T_{s}}^{t}\left|\eta_{4}(\tau)\right|^{2} d \tau+\frac{3}{2} \int_{T_{s}}^{t}\left|y_{1}(\tau)\right|^{2} d \tau+\frac{c_{\bar{e}}}{2}\right] \\
& \leq-\rho_{1} \varepsilon^{2} \int_{T_{s}}^{t}\left|\eta_{4}(\tau)\right|^{2} d \tau+\rho_{2} \varepsilon^{2} \hat{\varepsilon}^{2}(\varepsilon) \int_{T_{s}}^{t}\left|y_{1}(\tau)\right|^{2} d \tau+\rho_{3} \varepsilon^{2}
\end{aligned}
$$

for some positive constants $\rho_{1}, \rho_{2}$ and $\rho_{3}$. Using the smallgain condition (40), it follows that there exists a sufficiently small $\underline{\varepsilon}_{2}>0$ such that $\rho_{1}-\rho_{2} \hat{\varepsilon}^{2}(\varepsilon)>0$ and

$$
\int_{T_{s}}^{t}\left|\eta_{4}(\tau)\right|^{2} d \tau \leq \frac{1}{\rho_{1}-\rho_{2} \hat{\varepsilon}^{2}(\varepsilon)}\left(V^{\kappa}\left(T_{s}\right)+\rho_{3} \varepsilon^{2}\right)<\infty
$$

for all $\varepsilon \in\left(0, \min \left\{\varepsilon^{\star}, \underline{\varepsilon}_{2}\right\}\right)$. Setting $\underline{\varepsilon}:=\min \left\{\varepsilon^{\star}, \underline{\varepsilon}_{0}, \underline{\varepsilon}_{1}, \underline{\varepsilon}_{2}\right\}$, for any $\varepsilon \in(0, \underline{\varepsilon})$ and any $t \in\left[T_{s}, \infty\right)$ one obtains $\int_{T_{s}}^{t}\left|\eta_{4}(\tau)\right|^{2} d \tau<\infty$, hence the claim that $\eta_{4}(\cdot) \in \mathcal{L}_{2}$. This concludes the stability analysis, as all assumptions of Theorem 3.9 are now in place for the unique controller that remains active for all $t \geq T_{s}$.

\section{REDUCED-ORDER CONTROLLER}

A drawback of the multiple-model switching controller is its high dimensionality (it requires $3^{N}$ estimators, each with $4 N$ states), especially when the number of distinct excitation frequencies $N$ is large. Here, an alternative lowerdimensional scheme (comprising $3 N$ estimators, each with 4 states) is derived by exploiting the decoupled structure of the observers for $\hat{\zeta}_{o}$ and $\hat{\xi}_{1}$ given in (12) and (20). Since the lower-dimensional architecture fully preserves the properties of the multiple estimators, stability and convergence can be established along the lines of Section V. However, since the arguments would be more involved and less intuitive than the ones adopted for the larger-dimensional counterpart, in the interest of clarity this latter has been preferred in the presentation of the stability analysis.

For the reduced-order controller, instead of selecting the estimates $\hat{\theta}^{\sigma} \in \mathbb{R}^{2 N}$ based on a single performance index $J$, $N$ performance indexes $J_{n}$ are employed, each one used for selecting the corresponding parameter estimates $\hat{\theta}_{n}^{\sigma_{n}} \in \mathbb{R}^{2}$. For each excitation frequency, the new architecture of the switching controller is given as

$$
\begin{array}{ll}
\dot{\hat{\zeta}}_{o n}^{k}=S \hat{\zeta}_{o n}^{k}+\hat{\theta}_{n}^{k} u_{a n}^{\sigma_{n}}-\varepsilon g \tilde{y}_{n}^{k}, & \hat{\zeta}_{o n}^{k} \in \mathbb{R}^{2} \\
\dot{\hat{\theta}}_{n}^{k}=\operatorname{Proj}_{k}\left(\hat{\theta}_{n}^{k}, \varphi_{n}^{k}\left(\hat{\xi}_{1 n}, \tilde{y}_{n}^{k}\right)\right), & \hat{\theta}_{n}^{k}(0) \in \operatorname{int} \Theta_{k} \\
u_{a n}^{\sigma_{n}}=-\varepsilon \hat{\theta}_{n}^{\sigma_{n}} \hat{\zeta}_{o n}^{\sigma_{n}} & \\
\tilde{y}_{n}^{k}=g^{\top} \hat{\zeta}_{o n}^{k}-y_{n} & \\
y_{n}=y-\Sigma_{m=1, m \neq n}^{N} g^{\top} \hat{\zeta}_{o m}^{\sigma_{m}}, & k \in\{1,2,3\}, n \in \mathcal{N}
\end{array}
$$

where the control input and parameter estimates are given by $u_{a}=\operatorname{col}\left(u_{a 1}^{\sigma_{1}}, \ldots, u_{a N}^{\sigma_{N}}\right)$ and $\hat{\theta}^{\sigma}=\operatorname{col}\left(\hat{\theta}_{1}^{\sigma_{1} \top}, \ldots, \hat{\theta}_{N}^{\sigma_{N} \top}\right)$, and $\sigma_{n}(t)$ is a piecewise-constant signal taking value in $\{1,2,3\}$ determined by the switching mechanism based on $J_{n}^{k}$.
TABLE I

PARAMETER VECTORS AND SUBREGIONS OF THE PARAMETER SET

\begin{tabular}{|l|lc|c|}
\hline Frequency & \multicolumn{2}{|l|}{ True parameter vector $\theta$} & Convex set $\Theta_{i}$ \\
\hline \hline$\omega_{1}=1[\mathrm{rad} / \mathrm{s}]$ & $\theta_{1}=-0.2, \quad \theta_{2}=-0.6$ & $\Theta_{3}$ \\
\hline$\omega_{2}=3[\mathrm{rad} / \mathrm{s}]$ & $\theta_{1}=0.846, \quad \theta_{2}=0.231$ & $\Theta_{1}$ \\
\hline$\omega_{3}=5[\mathrm{rad} / \mathrm{s}]$ & $\theta_{1}=0.56, \quad \theta_{2}=0.72$ & $\Theta_{1}$ \\
\hline
\end{tabular}

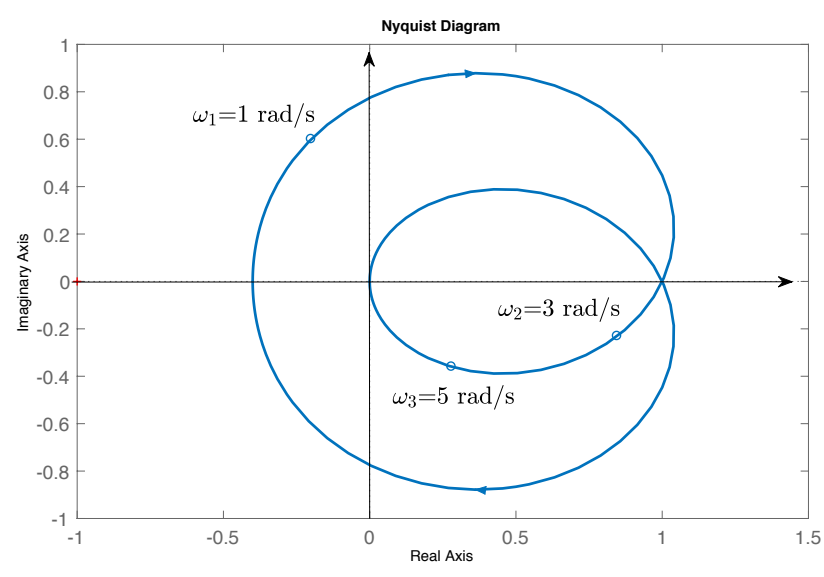

Fig. 6. Nyquist diagram of the stabilized plant model

Comparing the two realizations, one can easily verify that for any selection $\varsigma:=\left(\sigma_{1} \cdots \sigma_{N}\right)$ there always exists $i \in \mathcal{Q}$ such that $\mathcal{R}(i, n)=\sigma_{n}$ for all $n \in \mathcal{N}$, and vice versa. Therefore, the underlying principles of the multiple-model estimators and candidate controllers presented in SectionIV and this section are essentially equivalent. The only difference lies in the switching criteria where previously $\sigma(t):=$ $\arg \min _{i \in \mathcal{Q}}\left\{J_{h}^{i}(t)\right\}$ and $\sigma_{n}(t)=\arg \min _{k \in\{1,2,3\}}\left\{J_{n h}^{k}(t)\right\}$. While the two switching criteria may generate different switching signals, note that asymptotically accurate estimation of $\theta$ is not required for output stabilization. Thus, there exists more than one switching signals capable of selecting a stabilizing controller in finite time.

\section{ILLUSTRATIVE EXAMPLE}

A simulation study on an illustrative example is presented to validate the proposed methodology. Referring to Figure 1, let the stabilized plant model be described by the stable and non-minimum phase transfer function (assumed unknown)

$$
W(s)=\frac{2(s-1)}{s^{2}+2 s+5}
$$

with disturbance $d(t)=\sum_{i=1}^{3} \psi_{i} \sin \omega_{i} t, \psi_{i} \geq 0$.

Three distinct frequencies of excitation are considered, listed in Table I, together with the specific region of the covering $\bar{\Theta}$ for the corresponding parameter vectors $\theta_{n}$, $n=1,2,3$. The Nyquist diagram of $W(j \omega)$, displayed in Figure 6, shows that it is not possible to use a single controller of the type considered in [11], [12] to account for frequencies of the sinusoidal disturbance in the range given in Table I, as sign $\operatorname{Re}\left\{W\left(j \omega_{1}\right)\right\} \neq \operatorname{sign} \operatorname{Re}\left\{W\left(j \omega_{2}\right)\right\}$ and $\operatorname{sign} \operatorname{Im}\left\{W\left(j \omega_{1}\right)\right\} \neq \operatorname{sign} \operatorname{Im}\left\{W\left(j \omega_{2}\right)\right\}$. 
TABLE II

CONTROLLER INDEXES AND CORRESPONDING SUBREGIONS

\begin{tabular}{|c||c|c|c|c|c|c|c|c|c|}
\hline $\mathcal{Q}$ & 1 & 2 & 3 & 4 & 5 & 6 & 7 & 8 & 9 \\
\hline \hline$\hat{\theta}_{1}$ & $\Theta_{1}$ & $\Theta_{1}$ & $\Theta_{1}$ & $\Theta_{2}$ & $\Theta_{2}$ & $\Theta_{2}$ & $\Theta_{3}$ & $\Theta_{3}$ & $\Theta_{3}$ \\
\hline$\hat{\theta}_{2}$ & $\Theta_{1}$ & $\Theta_{2}$ & $\Theta_{3}$ & $\Theta_{1}$ & $\Theta_{2}$ & $\Theta_{3}$ & $\Theta_{1}$ & $\Theta_{2}$ & $\Theta_{3}$ \\
\hline
\end{tabular}

The controller and the multiple estimators are equipped with the same gains for all simulations: $\varepsilon=0.4, \rho=0.5$, $\beta=1, h=0.5, \delta_{1}=0.2, \delta_{2}=2$. The initial conditions of all states of the controller have been set at the origin, with the exception of the parameter estimates, whose initial conditions are chosen on the basis of each region of $\bar{\Theta}$ as

$$
\hat{\theta}_{n}^{i}(0)= \begin{cases}(11)^{\top} & \text { if } \hat{\theta}_{n}^{i}(0) \in \Theta_{1} \\ (-10)^{\top} & \text { if } \hat{\theta}_{n}^{i}(0) \in \Theta_{2} \\ (1-2)^{\top} & \text { if } \hat{\theta}_{n}^{i}(0) \in \Theta_{3}\end{cases}
$$

1) Exact order of the disturbance model: The first set of simulation results pertains to the case in which $\psi_{1}=\psi_{2}=$ $2, \psi_{3}=0$ and the order of the external model coincides with that of the disturbance model, namely $\hat{w} \in \mathbb{R}^{4}$ and $S=\operatorname{diag}\left(S_{1}, S_{2}\right)$ in System (5). In this case, $N=2$ and $3^{N}=9$ combinations are possible for the multiple model estimator. The labels of the estimators are detailed in Table II. The initial controller in the switching sequence is selected to be the one labeled as 5, whose estimates are constrained in subsets that do not contain the true parameters.

Simulation results are reported in Figure 7, which shows the time history of the output of the closed-loop system and the switching signal. Asymptotic regulation is achieved, and switchings terminate in about 20 [s]. It is noted that controller labeled as 4 is ultimately selected, whereas the controller corresponding to the true value of the parameters is the one labeled as 7 in Table II. Figure 8 shows the trajectory of the parameter estimates $\hat{\theta}_{n}^{4}(t), n=1,2$, which converge to constant values. The fact that $\lim _{t \rightarrow \infty} \tilde{\theta}_{n}^{4}(t) \neq 0$ implies that the regressor $\hat{\xi}_{1}(\cdot)$ is not persistently exciting. This is confirmed by Figure 9, which shows that $\hat{\xi}_{1}(t)$ vanishes asymptotically.

2) Over-parameterization of the disturbance model: The same controller of the previous case study is tested with $\psi_{1}=\psi_{3}=0$ and $\psi_{2}=2$, meaning that the controller now accounts for a larger number of distinct frequencies than the ones carried by the disturbance. Figure 10 indicates that lack of persistency of excitation of the disturbance (with respect to the order of the disturbance model) does not compromise the effectiveness of the proposed methodology.

3) Reduced-order controller: Finally, to show the effectiveness of the reduced-order multiple model estimator described in Section VI, we consider the case where disturbance signal contains all three excitation frequencies by letting $\psi_{i}=2, i=1,2,3$. The order of the external model is taken to match that of the disturbance model, that is, $\hat{w} \in \mathbb{R}^{6}$ and $S=\operatorname{diag}\left(S_{1}, S_{2}, S_{3}\right)$ in System (5). In this case, 27 estimators, each with 12 states, are needed to implement the original multiple-model estimator of Section IV. On the
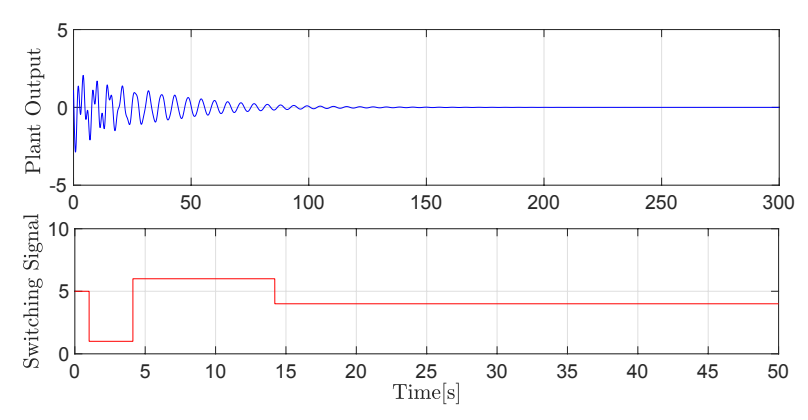

Fig. 7. Case study 1: Output trajectory, $y(t)$, and switching signal, $\sigma(t)$.
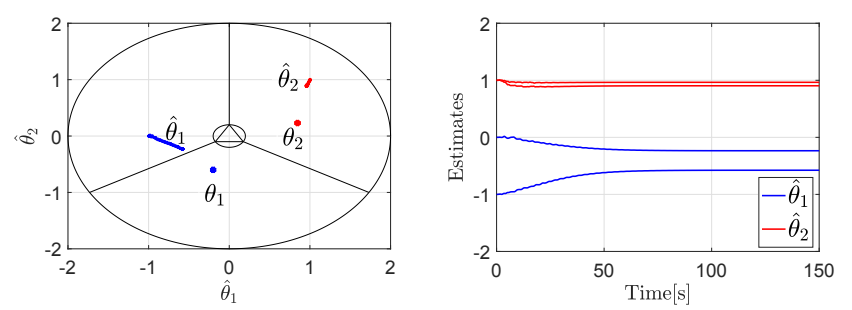

Fig. 8. Case study 1: Trajectories of the parameter estimates $\hat{\theta}_{n}^{4}(t)$ corresponding to the controller ultimately selected by the switching mechanism. other hand, the lower-dimensional architecture of Section VI requires only 9 estimators, each with 4 states, which is much more efficient in terms of computation cost. The result of the simulation is reported in Figure 11, which shows the asymptotically converging output $y(t)$ and the three switching signals, $\sigma_{n}(t)$, each converging in finite time.

\section{CONCLUDING REMARKS}

A methodology has been proposed to remove a longstanding requirement in $\mathrm{AFC}$, namely the necessity to impose SPR-like conditions on the transfer function at frequencies of interest. A drawback of the approach is the high dimensionality of the ensuing controller ( $14 N$ states for $N$ distinct excitation frequencies), which is an artifact of the multiple-model approach adopted herein to deal with a non-convex parameter set. This issue makes an extension to a MIMO setting an arduous task. Current investigations are pursuing alternative methods towards the design of a lower-dimensional controller, as well as the incorporation of frequency estimators in the control scheme to accommodate uncertain exosystem models.

\section{REFERENCES}

[1] M. Bodson, A. Sacks, and P. Khosla, "Harmonic generation in adaptive feedforward cancellation schemes," IEEE Transactions on Automatic Control, vol. 39, no. 9, pp. 1939-1944, 1994.

[2] W. Messner and M. Bodson, "Design of adaptive feedforward algorithms using internal model equivalence," International Journal of Adaptive Control and Signal Processing, vol. 9, no. 2, pp. 199-212, 1995.

[3] S. M. Kuo and D. R. Morgan, "Active noise control: a tutorial review," Proc. IEEE, vol. 87, no. 6, pp. 943-973, 1999.

[4] K. Ariyur and M. Krstic, "Feedback attenuation and adaptive cancellation of blade vortex interaction on a helicopter blade element," IEEE Transactions on Control Systems Technology, vol. 7, no. 5, pp. 596-605, 2002.

[5] D. Patt, D. Bernstein, J. Chandrasekar, P. Friedmann, and L. Liu, "Higher-harmonic-control algorithm for helicopter vibration reduction revisited," Journal of Guidance, Control, and Dynamics, vol. 28, no. 5, pp. 918-930, 2005. 


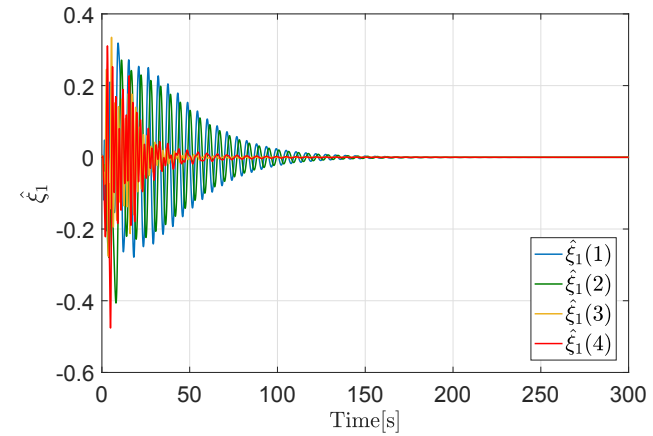

Fig. 9. Case study 1: Observer state $\hat{\xi}_{1}(t)$.
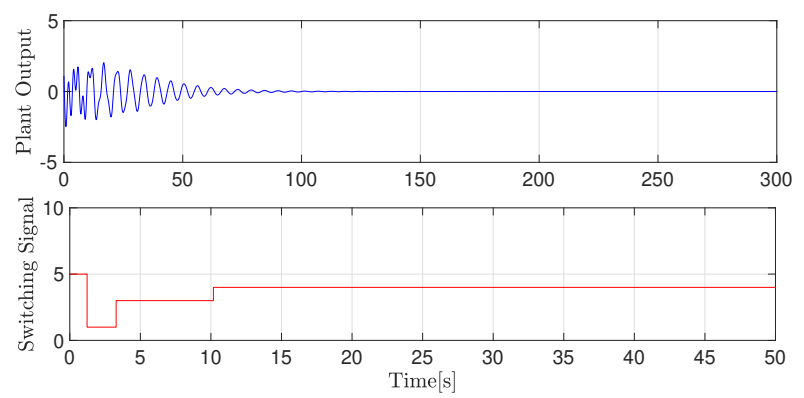

Fig. 10. Case study 2: Output trajectory, $y(t)$, and switching signal, $\sigma(t)$.

[6] X. Chen and M. Tomizuka, "A minimum parameter adaptive approach for rejecting multiple narrow-band disturbances with application to hard disk drives," IEEE Transactions on Control Systems Technology, vol. 20, no. 2, pp. 408-415, 2012.

[7] H. I. Basturk and M. Krstic, "Adaptive wave cancelation by acceleration feedback for ramp-connected air cushion-actuated surface effect ships," Automatica, vol. 49, no. 9, pp. 2591 - 2602, 2013.

[8] I. D. Landau, T.-B. Airimitoaie, A. Castellanos-Silva, and A. Constantinescu, Adaptive and Robust Active Vibration Control. Springer, 2017.

[9] P. Kokotovic, B. Riedle, and L. Praly, "On a stability criterion for continuous slow adaptation," Systems \& Control Letters, vol. 6, no. 1, pp. 7-14, 1985.

[10] S. Sastry and M. Bodson, Adaptive Control: Stability, Convergence, and Robustness. Prentice-Hall, 1989.

[11] R. Marino and P. Tomei, "Output Regulation for Unknown Stable Systems," IEEE Transactions on Automatic Control, vol. 60, no. 8, pp. 2213-2218, 2015.

[12] _ "Hybrid adaptive multi-sinusoidal disturbance cancellation," IEEE Transactions on Automatic Control, vol. 62, no. 8, pp. 40234030, 2017.

[13] H. I. Basturk and M. Krstic, "Adaptive sinusoidal disturbance cancellation for unknown LTI systems despite input delay," Automatica, vol. 58, pp. 131-138, 2015.

[14] S. Pigg and M. Bodson, "Adaptive Algorithms for the Rejection of Sinusoidal Disturbances Acting on Unknown Plants," IEEE Transactions on Control Systems Technology, vol. 18, no. 4, pp. 822-836, 2010.

[15] J. Chandrasekar, L. Liu, D. Patt, P. Friedmann, and D. Bernstein, "Adaptive Harmonic Steady-State Control for Disturbance Rejection," IEEE Transactions on Control Systems Technology, vol. 14, no. 6, pp. 993-1007, 2006.

[16] M. Kamaldar and J. B. Hoagg, "Adaptive harmonic steady-state control for rejection of sinusoidal disturbances acting on a completely unknown system," International Journal of Adaptive Control and Signal Processing, vol. 31, no. 9, pp. 1308-1326, 2017.

[17] C.-H. Chung and M.-S. Chen, "A robust adaptive feedforward control in repetitive control design for linear systems," Automatica, vol. 48, no. 1, pp. 183-190, 2012.

[18] S. Jafari and P. A. Ioannou, "Robust adaptive attenuation of unknown periodic disturbances in uncertain multi-input multi-output systems," Automatica, vol. 70, pp. 32-42, 2016.

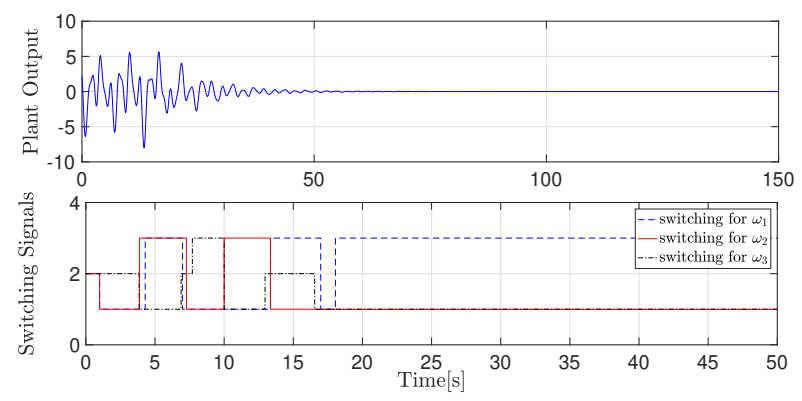

Fig. 11. Case study 3: Output trajectory, $y(t)$, and switching signals $\sigma_{n}(t)$, $n=1,2,3$, for the lower-dimensional controller.

[19] Y. Wang, G. Pin, A. Serrani, and T. Parisini, "Removing SPR-like conditions in adaptive feedforward control of uncertain systems," in Proceedings of the 55th IEEE Conference on Decision and Control, Melbourne (AUS), 2016, pp. 4728-4733.

[20] P. Ioannou and J. Sun, Robust Adaptive Control. Upper Saddle River, NJ: Prentice Hall, 1996.

[21] R. Middleton, G. Goodwin, D. Hill, and D. Mayne, "Design issues in adaptive control," IEEE Transactions on Automatic Control, vol. 33, no. 1 , pp. 50-58, 1988.

[22] A. S. Morse, D. Q. Mayne, and G. C. Goodwin, "Applications of hysteresis switching in parameter adaptive control," IEEE Transactions on Automatic Control, vol. 37, no. 9, pp. 1343-1354, 1992.

[23] K. Narendra and J. Balakrishnan, "Adaptive control using multiple models," IEEE Transactions on Automatic Control, vol. 42, no. 2, pp. 171-187, 1997.

[24] B. D. O. Anderson, T. S. Brinsmead, F. De Bruyne, J. Hespanha, D. Liberzon, and A. S. Morse, "Multiple model adaptive control. Part 1: Finite controller coverings," International Journal of Robust and Nonlinear Control, vol. 10, no. 11-12, pp. 909-929, 2000.

[25] J. Hespanha, D. Liberzon, S. A. Morse, B. D. O. Anderson, T. S. Brinsmead, and F. De Bruyne, "Multiple model adaptive control. Part 2: Switching," International Journal of Robust and Nonlinear Control, vol. 11, no. 5, pp. 479-496, 2001.

[26] A. Isidori, Nonlinear Control System II. Springer Verlag, 1999.

[27] Y. Lin, E. D. Sontag, and Y. Wang, "Input to state stabilizability for parametrized families of systems," International Journal of Robust and Nonlinear Control, vol. 5, no. 3, pp. 187-205, 1995.

[28] J. P. Hespanha, D. Liberzon, and A. Morse, "Hysteresis-based switching algorithms for supervisory control of uncertain systems," Automatica, vol. 39, no. 2, pp. $263-272,2003$.

[29] A. Kolmogorov and S. Fomin, Introductory Real Analysis. Dover Publications, 1975.

\section{APPENDIX}

\section{A. Derivation of the non-minimal realization (19)}

Define $\Psi(\tau):=\int_{0}^{\tau} \mathrm{e}^{-F_{\varepsilon} s} \boldsymbol{I}\left(u_{a}(s)\right) \mathrm{d} s \in \mathbb{R}^{2 N \times 2 N}$. Since $\frac{\mathrm{d}}{\mathrm{d} \tau} \Psi(\tau)=\mathrm{e}^{-F_{\varepsilon} \tau} \boldsymbol{I}\left(u_{a}(\tau)\right)$ and $\tilde{\boldsymbol{\theta}}(\tau) u_{a}(\tau)=\boldsymbol{I}\left(u_{a}(\tau)\right) \tilde{\theta}(\tau)$, integrating by parts, one obtains from (16)

$$
\begin{aligned}
& \tilde{y}_{1}(t)=\Gamma \mathrm{e}^{F_{\varepsilon} t} \int_{0}^{t} \frac{\mathrm{d}}{\mathrm{d} \tau} \Psi(\tau) \tilde{\theta}(\tau) \mathrm{d} \tau \\
& =\Gamma \mathrm{e}^{F_{\varepsilon} t}[\Psi(\tau) \tilde{\theta}(\tau)]_{0}^{t}-\Gamma \mathrm{e}^{F_{\varepsilon} t} \int_{0}^{t} \Psi(\tau) \dot{\tilde{\theta}}(\tau) \mathrm{d} \tau \\
& =\Gamma \mathrm{e}^{F_{\varepsilon} t} \int_{0}^{t} \mathrm{e}^{-F_{\varepsilon} \tau} \boldsymbol{I}\left(u_{a}(\tau)\right) \mathrm{d} \tau \tilde{\theta}(t)-\Gamma \mathrm{e}^{F_{\varepsilon} t} \int_{0}^{t} \Psi(\tau) \dot{\tilde{\theta}}(\tau) \mathrm{d} \tau .
\end{aligned}
$$

Thanks to the special structure of matrices $S$ and $\boldsymbol{I}\left(u_{a}\right)$, one obtains

$$
\begin{aligned}
{\left[\Gamma \mathrm{e}^{F_{\varepsilon}(t-\tau)} \boldsymbol{I}\left(u_{a}(\tau)\right)\right]^{\top} } & =\mathrm{e}^{S^{\top}(t-\tau)} \boldsymbol{I}\left(u_{a}(\tau)\right) \mathrm{e}^{-\varepsilon \Gamma^{\top} \Gamma(t-\tau)} \Gamma^{\top} \\
& =\mathrm{e}^{\left[S^{\top}-\varepsilon N G G^{\top}\right](t-\tau)} G u_{a}(\tau),
\end{aligned}
$$


where we have made used of the relations $\mathrm{e}^{-\varepsilon \Gamma^{\top} \Gamma(t-\tau)} \Gamma^{\top}=$ $\mathrm{e}^{-\varepsilon N(t-\tau)} G G^{\top} \Gamma^{\top}, \boldsymbol{I}\left(u_{a}(\tau)\right) G G^{\top}=G G^{\top} \boldsymbol{I}\left(u_{a}(\tau)\right)$ and $\boldsymbol{I}\left(u_{a}(\tau)\right) \Gamma^{\top}=G u_{a}(\tau)$. Defining $E_{\varepsilon}:=S-\varepsilon N G G$, one obtains

$\tilde{y}_{1}=\tilde{\theta}^{\top}(t) \int_{0}^{t} \mathrm{e}^{E_{\varepsilon}^{\top}(t-\tau)} G u_{a}(\tau) \mathrm{d} \tau-\Gamma \mathrm{e}^{F_{\varepsilon} t} \int_{0}^{t} \Psi(\tau) \dot{\tilde{\theta}}(\tau) \mathrm{d} \tau$.

The first term on the right-hand side has realization

$$
\dot{\xi}_{1}=E_{\varepsilon}^{\top} \xi_{1}+G u_{a}, \quad \tilde{y}_{1, a}=\tilde{\theta}^{\top}(t) \xi_{1} .
$$

For the second term, using $\dot{\tilde{\theta}}=\dot{\hat{\theta}}$, one obtains

$$
\begin{aligned}
& \Gamma \mathrm{e}^{F_{\varepsilon} t} \int_{0}^{t} \Psi(\tau) \dot{\tilde{\theta}}(\tau) \mathrm{d} \tau=\Gamma \mathrm{e}^{F_{\varepsilon} t} \int_{0}^{t} \int_{0}^{\tau} \mathrm{e}^{-F_{\varepsilon} s} \boldsymbol{I}\left(u_{a}(s)\right) \mathrm{d} s \dot{\hat{\theta}}(\tau) \mathrm{d} \tau \\
& =\Gamma \int_{0}^{t} \mathrm{e}^{F_{\varepsilon}(t-\tau)}\left[\int_{0}^{\tau} \mathrm{e}^{F_{\varepsilon}(\tau-s)} \boldsymbol{I}\left(u_{a}(s)\right) \mathrm{d} s\right] \dot{\hat{\theta}}(\tau) \mathrm{d} \tau \\
& =\Gamma \int_{0}^{t} \mathrm{e}^{F_{\varepsilon}(t-\tau)} \Xi(\tau) \dot{\hat{\theta}}(\tau) \mathrm{d} \tau
\end{aligned}
$$

where $\Xi(\tau):=\int_{0}^{\tau} \mathrm{e}^{F_{\varepsilon}(\tau-s)} \boldsymbol{I}\left(u_{a}(s)\right) \mathrm{d} s \in \mathbb{R}^{2 N \times 2 N}$ solves the matrix differential equation $\dot{\Xi}(t)=F_{\varepsilon} \Xi(t)+\boldsymbol{I}\left(u_{a}(t)\right)$ with initial condition $\Xi(0)=0_{2 N \times 2 N}$. Consequently, the second term admits the realization

$$
\dot{\xi}_{2}=F_{\varepsilon} \xi_{2}+\Xi \dot{\hat{\theta}}(t), \quad \dot{\Xi}=F_{\varepsilon} \Xi+\boldsymbol{I}\left(u_{a}\right), \quad \tilde{y}_{1, b}=\Gamma \xi_{2},
$$

hence (19) is achieved by letting $\tilde{y}_{1}=\tilde{y}_{1, a}-\tilde{y}_{1, b}$.

\section{B. Proof of Lemma 3.1}

Owing to the fact that $S-\varepsilon \boldsymbol{\theta} \boldsymbol{\theta}^{\top}$ and $\boldsymbol{I}_{o}(\theta, \varepsilon)$ are block diagonal matrices, the solution $P_{o}(\theta, \varepsilon)$ of (26) has a diagonal structure as well. Specifically, $P_{o}(\theta, \varepsilon)=\bigoplus_{n=1}^{N} P_{o, n}\left(\theta_{n}, \varepsilon\right)$, where $P_{o, n}\left(\theta_{n}, \varepsilon\right)$ is the solution of $P_{o, n}\left[\begin{array}{l}n=1 \\ \left.S_{n}-\varepsilon \theta_{n} \theta_{n}^{\top}\right]+\end{array}\right.$ $\left[S_{n}-\varepsilon \theta_{n} \theta_{n}^{\top}\right]^{\top} P_{o, n}=-\varepsilon \theta_{n}^{\top} \theta_{n} I_{2}, n \in \mathcal{N}$ and $I_{2}$ denotes a two-by-two the identity matrix. Let

$$
P_{o, n}=\left(\begin{array}{ll}
p_{n 1} & p_{n 2} \\
p_{n 3} & p_{n 4}
\end{array}\right)
$$

where

$$
\begin{aligned}
& p_{n 1}=\frac{\varepsilon^{2} \theta_{n 1}^{2} \theta_{n 2}^{2}+\varepsilon^{2} \theta_{n 2}^{4}+2 \varepsilon \omega_{n} \theta_{n 1} \theta_{n 2}+2 \omega_{n}^{2}}{2 \omega_{n}^{2}}, \\
& p_{n 2}=p_{n 3}=-\frac{\varepsilon\left(\varepsilon \theta_{n 1}^{3} \theta_{n 2}+\varepsilon \theta_{n 1} \theta_{n 2}^{3}+\omega_{n} \theta_{n 1}^{2}-\omega_{n} \theta_{n 2}^{2}\right)}{2 \omega_{n}^{2}}, \\
& p_{n 4}=\frac{\varepsilon^{2} \theta_{n 1}^{4}+\varepsilon^{2} \theta_{n 1}^{2} \theta_{n 2}^{2}-2 \varepsilon \omega_{n} \theta_{n 1} \theta_{n 2}+2 \omega_{n}^{2}}{2 \omega_{n}^{2}} .
\end{aligned}
$$

Since $\operatorname{det}\left(P_{o, n}\right)=\left(\varepsilon^{2}\left\|\theta_{n}\right\|^{4}+4 \omega_{n}^{2}\right) /\left(4 \omega_{n}^{2}\right)$, it follows that $\operatorname{det}\left(P_{o}(\theta, \varepsilon)\right)=\prod_{n=1}^{N} \operatorname{det}\left(P_{o, n}\left(\theta_{n}, \varepsilon\right)\right)>0$ for all $\left(\theta_{n}, \varepsilon\right) \in \Theta \times[0,+\infty)$. The spectrum of $P_{o}$ satisfies

$$
\operatorname{spec} P_{o}(\theta, 0)=\underbrace{\{1,1, \cdots, 1\}}_{2 N}
$$

for all $\theta_{n} \in \Theta$ and $n \in \mathcal{N}$. As a result, there exist $\bar{\varepsilon}>0$ and constants $0<c_{1}<c_{2} \leq c_{3}$ such that $c_{1} I \leq P_{o}(\theta, \varepsilon) \leq c_{2} I$ and $\left\|P_{o}(\theta, \varepsilon)\right\| \leq c_{3}$ for all $(\theta, \varepsilon) \in \Theta^{N} \times\left[0, \bar{\varepsilon}_{0}\right]$.

\section{Proof of Lemma 3.2}

Consider first the case of a single frequency, $N=1$. The solution of the Lyapunov equation

$$
P_{1}\left(\varepsilon_{1}\right) F_{1}+F_{1}^{\top} P_{1}\left(\varepsilon_{1}\right)=-\varepsilon_{1} I
$$

where

$$
F_{1}:=\left(\begin{array}{cc}
-\varepsilon_{1} & \omega_{1} \\
-\omega_{1} & 0
\end{array}\right), \quad P_{1}\left(\varepsilon_{1}\right)=\left(\begin{array}{cc}
1 & -\frac{\varepsilon_{1}}{2 \omega_{1}} \\
-\frac{\varepsilon_{1}}{2 \omega_{1}} & \frac{\varepsilon_{1}^{2}+2 \omega_{1}^{2}}{2 \omega_{1}^{2}}
\end{array}\right) .
$$

Note that $P_{1}\left(\varepsilon_{1}\right)$ is continuously differentiable with respect to $\varepsilon_{1}$ and positive definite for all $\varepsilon_{1} \in \mathbb{R}_{\geq 0}$, as $\operatorname{det}\left(P_{1}\left(\varepsilon_{1}\right)\right)=$ $\frac{\varepsilon_{1}^{2}+4 \omega_{1}^{2}}{4 \omega_{1}^{2}} \geq 1$. The eigenvalues of $P_{1}\left(\varepsilon_{1}\right)$ are found to be

$$
\begin{aligned}
& \lambda_{1,1}\left(\varepsilon_{1}\right)=\frac{\varepsilon_{1}^{2}+4 \omega_{1}^{2}+\sqrt{\varepsilon_{1}^{4}+4 \varepsilon_{1}^{2} \omega_{1}^{2}}}{4 \omega_{1}^{2}}, \\
& \lambda_{1,2}\left(\varepsilon_{1}\right)=\frac{\varepsilon_{1}^{2}+4 \omega_{1}^{2}-\sqrt{\varepsilon_{1}^{4}+4 \varepsilon_{1}^{2} \omega_{1}^{2}}}{4 \omega_{1}^{2}} .
\end{aligned}
$$

One can verify that $\frac{\partial \lambda_{1,1}\left(\varepsilon_{1}\right)}{\partial \varepsilon_{1}}$ is positive and $\lambda_{1,1}\left(\varepsilon_{1}\right)$ is a strictly increasing function of $\varepsilon_{1}$. Therefore, $c_{5,1}\left(\varepsilon_{1}\right):=$ $\lambda_{1,1}\left(\varepsilon_{1}\right)$ is a class- $\mathcal{N}$ function such that $P_{1}\left(\varepsilon_{1}\right) \leq c_{5,1}\left(\varepsilon_{1}\right) I$ for all $\varepsilon_{1} \in \mathbb{R}_{\geq 0}$. Similarly, the smallest eigenvalue $\lambda_{1,2}\left(\varepsilon_{1}\right)$ is a strictly decreasing function of $\varepsilon_{1}$; hence, letting $c_{4}=$ $\lim _{\varepsilon \rightarrow+\infty} \lambda_{1,2}\left(\varepsilon_{1}\right)=\frac{1}{2}$ yields $P_{1}\left(\varepsilon_{1}\right) \geq c_{4}$ for all $\varepsilon_{1} \in$ $\mathbb{R}_{\geq 0}$. Consequently, for the case of $N=1$, Lemma 3.2 is verified for $P_{F}(\varepsilon)$ and $Q_{F}(\varepsilon)$ by taking $\varepsilon=\varepsilon_{1}, F_{\varepsilon}=F_{1}$, $P_{F}(\varepsilon)=P_{1}, Q_{F}(\varepsilon)=-\varepsilon_{1} I, \bar{\varepsilon}_{1}=\varepsilon_{1}$ and $\lambda=1$. Next, let $N=2$. The solution of the Lyapunov equation

$$
P_{2}\left(\varepsilon_{2}\right) F_{2}+F_{2}^{\top} P_{2}\left(\varepsilon_{2}\right)=-\varepsilon_{2} I, F_{2}:=\left(\begin{array}{cc}
-\varepsilon_{2} & \omega_{2} \\
-\omega_{2} & 0
\end{array}\right)
$$

admits similar form and characteristics as $P_{1}\left(\varepsilon_{1}\right)$, that is, $P_{2}\left(\varepsilon_{2}\right)$ is positive definite for all $\varepsilon_{2} \in \mathbb{R}_{>0}$ and satisfies $c_{4} I \leq P_{2}\left(\varepsilon_{2}\right) \leq c_{5,2}\left(\varepsilon_{2}\right) I$, where $c_{5,2}\left(\varepsilon_{2}\right):=\lambda_{2,1}\left(\varepsilon_{2}\right)$ is a class $-\mathcal{N}$ function. For the Lyapunov equation (27), $F_{\varepsilon}$ takes the form

$$
F_{\varepsilon}=\left(\begin{array}{cc}
F_{1} & \varepsilon F_{x} \\
\varepsilon F_{x}^{\top} & F_{2}
\end{array}\right), \quad F_{x}:=\left(\begin{array}{cc}
-1 & 0 \\
0 & 0
\end{array}\right) .
$$

Since $P_{1}(\varepsilon)$ and $P_{2}(\varepsilon)$ are positive definite for all $\varepsilon \in \mathbb{R}_{>0}$, there exist a constant matrix $P_{x}$ and corresponding $\bar{\varepsilon}_{x}>0$ such that, for all $\varepsilon \in\left(0, \bar{\varepsilon}_{x}\right]$, the matrix

$$
P_{F}(\varepsilon):=\left(\begin{array}{cc}
P_{1} & \varepsilon P_{x} \\
\varepsilon P_{x}^{\top} & P_{2}
\end{array}\right)
$$

is positive definite. This yields the matrix $Q_{F}(\varepsilon)$

$$
Q_{F}(\varepsilon)=P_{F}(\varepsilon) F_{\varepsilon}+F_{\varepsilon}^{\top} P_{F}(\varepsilon):=\left(\begin{array}{ll}
q_{1} & q_{2} \\
q_{3} & q_{4}
\end{array}\right)
$$


where

$$
\begin{aligned}
& q_{1}=P_{1} F_{1}+\varepsilon^{2} P_{x} F_{x}^{\top}+F_{1}^{\top} P_{1}+\varepsilon^{2} F_{x} P_{x}^{\top}, \\
& q_{2}=q_{3}^{\top}=\varepsilon P_{1} F_{x}+\varepsilon P_{x} F_{2}+\varepsilon F_{1}^{\top} P_{x}+\varepsilon F_{x} P_{2}, \\
& q_{4}=P_{2} F_{2}+\varepsilon^{2} P_{x}^{\top} F_{x}+F_{2}^{\top} P_{2}+\varepsilon^{2} F_{x}^{\top} P_{x} .
\end{aligned}
$$

Since by assumption $\omega_{1} \neq \omega_{2}$, it follows that $\operatorname{spec} F_{1}^{\top} \cap$ $\operatorname{spec} F_{2}=\emptyset$. As a result, there exists a unique solution $P_{x}$ of the Sylvester equation $P_{x} F_{2}+F_{1}^{\top} P_{x}=-P_{1} F_{x}-F_{x} P_{2}$ verifying $q_{2}=q_{3}^{\top}=0$. Using the Lyapunov equations (47) and (48), one obtains

$$
\begin{aligned}
& q_{1}=-\varepsilon I+\varepsilon^{2}\left(P_{x} F_{x}^{\top}+F_{x} P_{x}^{\top}\right) \\
& q_{4}=-\varepsilon I+\varepsilon^{2}\left(P_{x}^{\top} F_{x}+F_{x}^{\top} P_{x}\right)
\end{aligned}
$$

from which it follows that there exist constants $\bar{\varepsilon}_{1} \in\left(0, \bar{\varepsilon}_{x}\right]$ and $\lambda \in \mathbb{R}_{>0}$, such that $P_{F}(\varepsilon) F_{\varepsilon}+F_{\varepsilon}^{\top} P_{F}(\varepsilon)=Q_{F}(\varepsilon) \leq$ $-\lambda \varepsilon I$ for all $\varepsilon \in\left(0, \bar{\varepsilon}_{1}\right]$. Moreover, exploiting the special structure of $P_{F}(\varepsilon)$ in (49), boundedness of the determinant and the eigenvalues of the matrix $P_{F}(\varepsilon)$ are easily verified. This completes the proof for the case $N=2$. The proof for $N>2$ proceeds analogously by constructing the solution

$$
P_{F}(\varepsilon):=\left(\begin{array}{ccccc}
P_{1} & P_{x, 1} & P_{x, 2} & \cdots & P_{x, N-1} \\
P_{x, 1}^{\top} & P_{2} & P_{y, 2} & \cdots & P_{y, N-2} \\
\vdots & \vdots & \ddots & \ddots & \vdots \\
P_{x, N-1}^{\top} & P_{y, N-2}^{\top} & \cdots & \cdots & P_{N}
\end{array}\right) .
$$

\section{Proof of Proposition 3.3}

First, the following preliminary result is needed:

Property 1: The gradient with respect to $\theta$ of the quadratic form $Q_{o}(w):=w^{\top} P_{o}(\theta, \varepsilon) w, w \in \mathbb{R}^{2 N}$, satisfies $\nabla_{\theta} Q_{o}(w)=\varepsilon\left(w^{\top} R_{1}(\theta, \varepsilon) w \cdots w^{\top} R_{2 N}(\theta, \varepsilon) w\right)$ for some matrix-valued functions $R_{j}:(\theta, \varepsilon) \rightarrow \mathbb{R}^{2 N \times 2 N}$, which are continuous and bounded for $(\theta, \varepsilon) \in \Theta^{N} \times\left(0, \bar{\varepsilon}_{0}\right]$.

Evaluating the derivative of the Lyapunov function candidate $V_{1}\left(t, \hat{\zeta}_{o}\right):=\hat{\zeta}_{o}^{\top} P_{o}(\hat{\theta}(t), \varepsilon) \hat{\zeta}_{o}$ along trajectories of System (23), yields, for all $\left(\hat{\theta}_{n}(\cdot), \varepsilon\right) \in \hat{\Theta} \times\left(0, \bar{\varepsilon}_{0}\right]$

$$
\begin{aligned}
\dot{V}_{1}= & -\hat{\zeta}_{o}^{\top} \boldsymbol{I}_{o}(\hat{\theta}, \varepsilon) \hat{\zeta}_{o}+\frac{\partial \hat{\zeta}_{o}^{\top} P_{o} \hat{\zeta}_{o}}{\partial \hat{\theta}} \dot{\hat{\theta}}+2 \varepsilon \hat{\zeta}_{o}^{\top} P_{o} \Gamma^{\top} \nu_{1} \\
\leq & -\frac{\varepsilon}{2} N \delta_{1}^{2}\left\|\hat{\zeta}_{o}\right\|^{2}-\frac{\varepsilon}{2} \sum_{n=1}^{N} \hat{\zeta}_{o n}^{\top} \hat{\theta}_{n}^{\top} \hat{\theta}_{n} \hat{\zeta}_{o n}+\varepsilon^{3} \rho \ell_{1}\left\|\hat{\zeta}_{o}\right\|^{2} \\
& +2 \varepsilon c_{3} \sqrt{N}\left\|\hat{\zeta}_{o}\right\|\left|\nu_{1}\right|
\end{aligned}
$$

where we have made use of Lemma 3.1, Property 1 and Definition 3.1, having denoted by $\ell_{1}$ the quantity

$$
\ell_{1}:=\sum_{n=1}^{N} \max _{\hat{\theta}_{n} \in \hat{\Theta}, \varepsilon \in\left(0, \overline{\varepsilon_{0}}\right]}\left\{\left\|R_{2 n-1}(\hat{\theta}, \varepsilon)\right\|+\left\|R_{2 n}(\hat{\theta}, \varepsilon)\right\|\right\},
$$

and $\delta_{1}$ is defined in (7). Adding and subtracting $\varepsilon\left\|\eta_{1}\right\|^{2} / 2$ in (50) and using $\left\|\eta_{1}\right\|^{2} \leq \sum_{n=1}^{N} \hat{\zeta}_{\text {on }}^{\top} \hat{\theta}_{n}^{\top} \hat{\theta}_{n} \hat{\zeta}_{\text {on }}$, yield

$$
\begin{aligned}
\dot{V}_{1} \leq & -\frac{\varepsilon}{2} N \delta_{1}^{2}\left\|\hat{\zeta}_{o}\right\|^{2}-\frac{\varepsilon}{2}\left\|\eta_{1}\right\|^{2}+\varepsilon^{3} \rho \ell_{1}\left\|\hat{\zeta}_{o}\right\|^{2} \\
& +2 \varepsilon c_{3} \sqrt{N}\left\|\hat{\zeta}_{o}\right\|\left|\nu_{1}\right| .
\end{aligned}
$$

Making use of Young's inequality in the form $2|a b| \leq \ell_{2}^{2} a^{2}+$ $\frac{1}{\ell_{2}^{2}} b^{2}$ for the last term in (51) and choosing $\ell_{2}:=\frac{2}{\delta_{1}}$ yield

$$
\dot{V}_{1} \leq-\varepsilon\left(\frac{\delta_{1}^{2}}{4} N-\varepsilon^{2} \rho \ell_{1}\right)\left\|\hat{\zeta}_{o}\right\|^{2}-\frac{\varepsilon}{2}\left\|\eta_{1}\right\|^{2}+\varepsilon \ell_{2}^{2} c_{3}^{2}\left|\nu_{1}\right|^{2} .
$$

Letting $\varepsilon_{1}^{\star}:=\min \left\{\bar{\varepsilon}_{0}, \frac{\delta_{1} \sqrt{N}}{2 \sqrt{2 \rho \ell_{1}}}\right\}$, it follows that, for all $\left(\hat{\theta}_{n}(\cdot), \varepsilon\right) \in \hat{\Theta}_{\varepsilon} \times\left(0, \varepsilon_{1}^{\star}\right)$,

$$
\dot{V}_{1} \leq-\frac{\varepsilon}{2} \lambda_{1}(\varepsilon)\left\|\hat{\zeta}_{o}\right\|^{2}-\frac{\varepsilon}{2}\left\|\eta_{1}\right\|^{2}+\frac{\varepsilon}{2} 2 \ell_{2}^{2} c_{3}^{2}\left|\nu_{1}\right|^{2}
$$

where $\lambda_{1}(\varepsilon):=\frac{\delta_{1}^{2}}{2} N-2 \varepsilon^{2} \rho \ell_{1}>\frac{\delta_{1}^{2}}{4} N>0$. This shows that for any $\varepsilon \in\left(0, \varepsilon_{1}^{\star}\right)$, System (23) is strictly dissipative with respect to the supply rate $q_{1}\left(\nu_{1}, \eta_{1}\right)=\gamma_{1}^{\star 2}\left|\nu_{1}\right|^{2}-\left\|\eta_{1}\right\|^{2}$, $\gamma_{1}^{\star}:=\sqrt{2} \ell_{2} c_{3}$, with storage function $W_{1}\left(t, \hat{\zeta}_{o}\right):=\frac{2}{\varepsilon} V_{1}\left(t, \hat{\zeta}_{o}\right)$ satisfying $\dot{W}_{1} \leq-\lambda_{1}(\varepsilon)\left\|\hat{\zeta}_{o}\right\|^{2}-\left\|\eta_{1}\right\|^{2}+\gamma_{1}^{\star^{2}}\left|\nu_{1}\right|^{2}$.

\section{E. Proof of Lemma 3.6}

As in the proof of Lemma 3.1, the block diagonal structure of $E_{\varepsilon}$ results in a block diagonal solution $P_{E}(\varepsilon)=$ $\bigoplus_{n=1}^{N} P_{E, n}(\varepsilon)$, where

$$
P_{E, n}(\varepsilon)=\left(\begin{array}{cc}
1 & -\frac{\varepsilon N}{2 \omega_{n}} \\
-\frac{\varepsilon N}{2 \omega_{n}} & \frac{(\varepsilon N)^{2}+2 \omega_{n}^{2}}{2 \omega_{n}^{2}}
\end{array}\right)
$$

is the solution of the Lyapunov equation $P_{E, n}(\varepsilon)\left(S_{n}-\right.$ $\left.\varepsilon N g g^{\top}\right)+\left(S_{n}-\varepsilon N g g^{\top}\right)^{\top} P_{E, n}(\varepsilon)=-\varepsilon N I$. The entries of $P_{E, n}(\varepsilon)$ are continuously differentiable functions of $\varepsilon$ and positive definite for all $\varepsilon \in \mathbb{R}_{\geq 0}$ as $\operatorname{det}\left(P_{E, n}(\varepsilon)\right)=$ $\frac{(\varepsilon N)^{2}+4 \omega_{n}^{2}}{4 \omega_{n}^{2}} \geq 1$, and the eigenvalues of $P_{E, n}(\varepsilon)$ are

$$
\begin{aligned}
& \sigma_{n, 1}(\varepsilon)=\frac{\varepsilon^{2} N^{2}+4 \omega_{n}^{2}+\sqrt{\varepsilon^{4} N^{4}+4 \varepsilon^{2} N^{2} \omega_{n}^{2}}}{4 \omega_{n}^{2}}, \\
& \sigma_{n, 2}(\varepsilon)=\frac{\varepsilon^{2} N^{2}+4 \omega_{n}^{2}-\sqrt{\varepsilon^{4} N^{4}+4 \varepsilon^{2} N^{2} \omega_{n}^{2}}}{4 \omega_{n}^{2}} .
\end{aligned}
$$

One can verify that $\frac{\partial \sigma_{n, 1}(\varepsilon)}{\partial \varepsilon}$ is positive and the largest eigenvalue $\sigma_{n, 1}(\varepsilon)$ is a strictly increasing function of $\varepsilon$. Therefore, there exists a class- $\mathcal{N}$ function $c_{7, n}(\varepsilon)=\sigma_{n, 1}(\varepsilon)$ such that $P_{E, n}(\varepsilon) \leq c_{7, n}(\varepsilon) I$ for all $\varepsilon \in \mathbb{R}_{\geq 0}$ and all $n \in \mathcal{N}$. Similarly, it is possible to show that the smallest eigenvalue $\sigma_{n, 2}(\varepsilon)$ is a strictly decreasing function of $\varepsilon$, hence there exists a constant $c_{6}=\lim _{\varepsilon \rightarrow+\infty} \sigma_{n, 2}(\varepsilon)=\frac{1}{2}$ satisfying $P_{E, n}(\varepsilon) \geq c_{6}$ for all $\varepsilon \in \mathbb{R}_{\geq 0}$ and all $n \in \mathcal{N}$. Owing to the block diagonal structure of $P_{E, n}(\varepsilon)$, it follows that $\operatorname{eig}\left(P_{E}(\varepsilon)\right)=\left(\sigma_{1,1}, \sigma_{1,2}, \sigma_{2,1}, \sigma_{2,2}, \cdots \sigma_{N, 1}, \sigma_{N, 2}\right)$. As a result, $P_{E, n}(\varepsilon)$ is positive definite and bounded (in the sense of positive semi-definiteness) by $c_{6} I$ and $\max _{n \in \mathcal{N}}\left\{c_{7, n}(\varepsilon)\right\} I . \triangleleft$

\section{F. Proof of Proposition 5.1}

The proof proceeds by contradiction. Without loss of generality, assume that a non-terminating switching takes place between two controllers, labelled as 1 and 2. Since the switching sequence does not stop, there exists a subsequence $\left\{t_{m}\right\}_{m=0}^{\infty} \subset\left\{T_{m}\right\}_{m=0}^{\infty}$ such that

$$
\sigma(t)=\left\{\begin{array}{ll}
1, & t \in\left[t_{2 m}, t_{2 m+1}\right) \\
2, & t \in\left[t_{2 m+1}, t_{2 m+2}\right)
\end{array} \quad \forall m \in\{0,1,2, \cdots\} .\right.
$$


Then, according to (36), one obtains

$$
J^{1}\left(t_{2 m}\right)<J^{2}\left(t_{2 m}\right)-h, J^{1}\left(t_{2 m+1}\right)>J^{2}\left(t_{2 m+1}\right)+h
$$

which, rearranging terms, yields

$$
\begin{aligned}
& {\left[J^{1}\left(t_{2 m+1}\right)-J^{1}\left(t_{2 m}\right)\right]-\left[J^{2}\left(t_{2 m+1}\right)-J^{2}\left(t_{2 m}\right)\right]=} \\
& \beta \int_{t_{2 m}}^{t_{2 m+1}}\left|\tilde{y}^{1}(\tau)\right|^{2} d \tau-\beta \int_{t_{2 m}}^{t_{2 m+1}}\left|\tilde{y}^{2}(\tau)\right|^{2} d \tau>2 h
\end{aligned}
$$

with $m \in\{1,2 \cdots\}$ and $\tilde{y}^{i}, i=1,2$ is given in (37). Substituting the inequalities

$$
\begin{aligned}
& \left|\tilde{y}^{1}\right|^{2} \leq\left|\hat{\xi}_{1}^{\top} \tilde{\theta}^{1}\right|^{2}+\left|y_{1}\right|^{2}+2\left|\hat{\xi}_{1}^{\top} \tilde{\theta}^{1}\right|\left|y_{1}\right| \leq 2\left|\hat{\xi}_{1}^{\top} \tilde{\theta}^{2}\right|^{2}+2\left|y_{1}\right|^{2} \\
& \left|\tilde{y}^{2}\right|^{2} \geq\left|\hat{\xi}_{1}^{\top} \tilde{\theta}^{2}\right|^{2}+\left|y_{1}\right|^{2}-2\left|\hat{\xi}_{1}^{\top} \tilde{\theta}^{2}\right|\left|y_{1}\right| \geq \frac{1}{2}\left|\hat{\xi}_{1}^{\top} \tilde{\theta}^{2}\right|^{2}-\left|y_{1}\right|^{2}
\end{aligned}
$$

into (52), it follows that

$$
2 h<\beta \int_{t_{2 m}}^{t_{2 m+1}} 2\left|\hat{\xi}_{1}^{\top} \tilde{\theta}^{1}(\tau)\right|^{2}-\frac{1}{2}\left|\hat{\xi}_{1}^{\top} \tilde{\theta}^{2}(\tau)\right|^{2}+3\left|y_{1}(\tau)\right|^{2} d \tau
$$

where $y_{1}$ is defined in (22). By virtue of the small gain interconnection in (40), there exist positive constants $\mu_{1}$ and $\mu_{2}$ satisfying

$$
\int_{t_{2 m}}^{t_{2 m+1}} \hat{\xi}_{1}^{\top} \hat{\xi}_{1}\left(\mu_{1}\left\|\tilde{\theta}^{1}(\tau)\right\|^{2}-\left\|\tilde{\theta}^{2}(\tau)\right\|^{2}\right) d \tau>\mu_{2} h .
$$

Similarly, in the interval $\left[t_{2 m+1}, t_{2 m+2}\right)$

$$
\int_{t_{2 m+1}}^{t_{2 m+2}} \hat{\xi}_{1}^{\top} \hat{\xi}_{1}\left(\mu_{1}\left\|\tilde{\theta}^{2}(\tau)\right\|^{2}-\left\|\tilde{\theta}^{1}(\tau)\right\|^{2}\right) d \tau>\mu_{2} h .
$$

Since $m$ can be arbitrarily large and the vectors $\tilde{\theta}^{i}, i=1,2$ are norm-bounded from above, (54) and (55) imply that

$$
\min \left\{\int_{t_{2 m}}^{t_{2 m+1}} \hat{\xi}_{1}(\tau)^{\top} \hat{\xi}_{1}(\tau) d \tau, \int_{t_{2 m+1}}^{t_{2 m+2}} \hat{\xi}_{1}(\tau)^{\top} \hat{\xi}_{1}(\tau) d \tau\right\}>\mu_{3} h
$$

for some constant $\mu_{3}>0$. Next, consider the storage function $V^{\kappa}\left(\tilde{\theta}^{\kappa}\right)=\frac{1}{2}\left\|\tilde{\theta}^{\kappa}\right\|^{2}$, where $\tilde{\theta}^{\kappa}:=\hat{\theta}^{\kappa}-\theta$, and $\kappa$ denotes the label of the estimator whose parameter estimates $\hat{\theta}_{n}^{\kappa}$ are all constrained in the region that contains the true parameter vector $\theta_{n}$, i.e. $\theta_{n} \in \Theta_{\mathcal{R}(\kappa, n)}$, for all $n \in \mathcal{N}$. Evaluating the derivative of $V^{\kappa}$ along the trajectories of the parameter estimates governed by (33) and (34) on the time interval $\left[T_{s}, \infty\right)$ yields

$$
\dot{V}^{\kappa}=-\rho \varepsilon^{2} \tilde{\theta}^{\kappa \top} \frac{\hat{\xi}_{1} \tilde{y}^{\kappa}}{m_{\kappa}^{2}}-\tilde{\theta}^{\kappa \top} \mathcal{I}_{\kappa}\left(\hat{\theta}^{\kappa}, \varphi\right) \varphi \leq-\rho \varepsilon^{2} \tilde{\theta}^{\kappa \top} \frac{\hat{\xi}_{1} \tilde{y}^{\kappa}}{m_{\kappa}^{2}}
$$

where the term $\tilde{\theta}^{\kappa \top} \mathcal{I}_{\kappa}\left(\hat{\theta}^{\kappa}, \varphi^{\kappa}\right) \varphi^{\kappa}$ introduced by the projection is non-negative, since the true parameter vectors $\theta_{n} \in \Theta_{\mathcal{R}(\kappa, n)}$ for all $n \in \mathcal{N}$. Recalling that $\tilde{y}^{\kappa}=\hat{\xi}_{1}^{\top} \tilde{\theta}^{\kappa}+y_{1}$ and integrating both sides of (57) from $t_{2 m}$ to $t_{2 m+2}$ yields

$$
\begin{aligned}
V^{\kappa}\left(t_{2 m+2}\right)-V^{\kappa}\left(t_{2 m}\right) \leq \frac{\rho \varepsilon^{2}}{2} \int_{t_{2 m}}^{t_{2 m+2}}\left|y_{1}(\tau)\right|^{2} d \tau \\
-\frac{\rho \varepsilon^{2}}{2\left\|m_{\kappa}\right\|_{\infty}^{2}} \int_{t_{2 m}}^{t_{2 m+2}}\left|\hat{\xi}_{1}(\tau)^{\top} \tilde{\theta}^{\kappa}(\tau)\right|^{2} d \tau .
\end{aligned}
$$

Using the small-gain connection (40), one obtains

$$
\begin{aligned}
& \int_{t_{2 m}}^{t_{2 m+2}}\left|y_{1}(\tau)\right|^{2} d \tau \leq \hat{\varepsilon}^{2}(\varepsilon) \int_{t_{2 m}}^{t_{2 m+2}} \hat{\xi}_{1}^{\top} \hat{\xi}_{1}\left\|\tilde{\theta}^{\sigma}(\tau)\right\|^{2} d \tau \\
\leq & \hat{\varepsilon}^{2}(\varepsilon) \int_{t_{2 m}}^{t_{2 m+1}} \hat{\xi}_{1}^{\top} \hat{\xi}_{1}\left\|\tilde{\theta}^{1}(\tau)\right\|^{2} d \tau+\hat{\varepsilon}^{2}(\varepsilon) \int_{t_{2 m+1}}^{t_{2 m+2}} \hat{\xi}_{1}^{\top} \hat{\xi}_{1}\left\|\tilde{\theta}^{2}(\tau)\right\|^{2} d \tau
\end{aligned}
$$

which implies that

$$
\begin{aligned}
& V^{\kappa}\left(t_{2 m+2}\right)-V^{\kappa}\left(t_{2 m}\right) \\
& \leq-\frac{\rho \varepsilon^{2}}{2} \int_{t_{2 m}}^{t_{2 m+1}} \hat{\xi}_{1}^{\top} \hat{\xi}_{1}\left(\frac{\left\|\tilde{\theta}^{\kappa}(\tau)\right\|^{2}}{\left\|m_{\kappa}\right\|_{\infty}^{2}}-\hat{\varepsilon}^{2}(\varepsilon)\left\|\tilde{\theta}^{1}(\tau)\right\|^{2}\right) d \tau \\
& \quad-\frac{\rho \varepsilon^{2}}{2} \int_{t_{2 m+1}}^{t_{2 m+2}} \hat{\xi}_{1}^{\top} \hat{\xi}_{1}\left(\frac{\left\|\tilde{\theta}^{\kappa}(\tau)\right\|^{2}}{\left\|m_{\kappa}\right\|_{\infty}^{2}}-\hat{\varepsilon}^{2}(\varepsilon)\left\|\tilde{\theta}^{2}(\tau)\right\|^{2}\right) d \tau .
\end{aligned}
$$

For the time being, assume that the controller $\kappa$ takes part in the non-terminating switching sequence, i.e., $\kappa \in\{1,2\}$. Without loss of generality, assume $\kappa=1$. Consequently,

$$
\begin{aligned}
& V^{\kappa}\left(t_{2 m+2}\right)-V^{\kappa}\left(t_{2 m}\right) \\
& \leq-\frac{\rho \varepsilon^{2}}{2} \int_{t_{2 m+1}}^{t_{2 m+2}} \hat{\xi}_{1}^{\top} \hat{\xi}_{1}\left(\frac{\left\|\tilde{\theta}^{\kappa}(\tau)\right\|^{2}}{\left\|m_{\kappa}\right\|_{\infty}^{2}}-\hat{\varepsilon}^{2}(\varepsilon)\left\|\tilde{\theta}^{2}(\tau)\right\|^{2}\right) d \tau .
\end{aligned}
$$

Let $\varepsilon>0$ be small enough so that $\hat{\varepsilon}^{2}(\varepsilon)\left\|m_{\kappa}\right\|_{\infty}^{2}<1$. Using the fact that (56) implies that $\hat{\xi}_{1}(t)$ is bounded away from zero, it is seen that $V^{\kappa}(t)$ and $\left\|\tilde{\theta}^{\kappa}(t)\right\|^{2}$ will keep decreasing until the left hand side of (59) is equal to zero. This implies that there exists an integer $m_{0}$ such that

$$
\begin{aligned}
\left\|\tilde{\theta}^{\kappa}(t)\right\|^{2} & \leq \hat{\varepsilon}^{2}(\varepsilon)\left\|m_{\kappa}\right\|_{\infty}^{2} \max \left\{\left\|\tilde{\theta}^{2}(t)\right\|^{2}\right\} \\
& \leq 4 \hat{\varepsilon}^{2}(\varepsilon) N \delta_{2}^{2}\left\|m_{\kappa}\right\|_{\infty}^{2}
\end{aligned}
$$

for all $t \geq t_{2 m_{0}}$. Since not all $\theta_{n}$ belong to $\Theta_{\mathcal{R}(2, n)}$, the bound $\left\|\tilde{\theta}^{2}\right\|^{2} \geq N \delta_{1}^{\prime 2}$ holds for some $\delta_{1}^{\prime}>0$. Therefore, for all $\varepsilon>0$ satisfying $4 \mu_{1} \hat{\varepsilon}^{2}(\varepsilon) \delta_{2}^{2}\left\|m_{\kappa}\right\|_{\infty}^{2}-\delta_{1}^{\prime 2}<0$, where $\mu_{1}$ is the constant defined in (54) and (55), one obtains $\mu_{1}\left\|\tilde{\theta}^{1}(t)\right\|-\left\|\tilde{\theta}^{2}(t)\right\|<0$ for all $t \geq t_{2 m_{0}}$, which violates (54). Similarly, if $\kappa=2$, (55) does not hold for $t>t_{2 m_{0}}$. This contradiction implies that there exists a finite time instant $T_{s} \geq t_{2 m_{0}}$ at witch the switching stops.

Next, it will be shown that $\kappa \in\{1,2\}$ for sufficient small $\varepsilon>0$. Assume $\kappa \notin\{1,2\}$ and consider the auxiliary variable $\tilde{J}(t):=J^{\kappa}(t)-J^{2}(t)$. Then, for any $m \geq m_{0}$

$$
\tilde{J}\left(t_{2 m+1}\right)=\tilde{J}\left(t_{2 m_{0}}\right)+\beta \int_{t_{2 m_{0}}}^{t_{2 m+1}}\left|\tilde{y}^{\kappa}(\tau)\right|^{2}-\left|\tilde{y}^{2}(\tau)\right|^{2} d \tau .
$$

Following the arguments used to derive (53), one obtains

$$
\begin{aligned}
& \tilde{J}\left(t_{2 m+1}\right) \leq \tilde{J}\left(t_{2 m_{0}}\right) \\
& +\beta \int_{t_{2 m_{0}}}^{t_{2 m+1}} 2\left|\hat{\xi}_{1}^{\top} \tilde{\theta}^{\kappa}(\tau)\right|^{2}-\frac{1}{2}\left|\hat{\xi}_{1}^{\top} \tilde{\theta}^{2}(\tau)\right|^{2}+3\left|y_{1}(\tau)\right|^{2} d \tau .
\end{aligned}
$$


By virtue of the small-gain interconnection (29)

$$
\begin{aligned}
& \beta \int_{t_{2 m_{0}}}^{t_{2 m+1}}\left|y_{1}(\tau)\right|^{2} d \tau \leq \beta \sum_{j=m_{0}}^{m} \int_{t_{2 j}}^{t_{2 j+1}} \hat{\varepsilon}^{2}(\varepsilon)\left|\hat{\xi}_{1}^{\top} \tilde{\theta}^{1}(\tau)\right|^{2} d \tau \\
& +\beta \sum_{j=m_{0}}^{m-1} \int_{t_{2 j+1}}^{t_{2 j+2}} \hat{\varepsilon}^{2}(\varepsilon)\left|\hat{\xi}_{1}^{\top} \tilde{\theta}^{2}(\tau)\right|^{2} d \tau \leq \beta \int_{t_{2 m_{0}}}^{t_{2 m+1}} \hat{\varepsilon}^{2}(\varepsilon) \delta_{3}\left|\hat{\xi}_{1}^{\top} \tilde{\theta}^{2}(\tau)\right|^{2} d \tau
\end{aligned}
$$

where we have use the fact that, since $\kappa \notin\{1,2\}$, there exists $\delta_{3}>1$ such that $\left\|\tilde{\theta}^{1}(\tau)\right\|^{2} \leq \delta_{3}\left\|\tilde{\theta}^{2}(\tau)\right\|^{2}$. Applying (61) to (60) yields

$\tilde{J}\left(t_{2 m+1}\right) \leq \tilde{J}\left(t_{2 m_{0}}\right)+\beta \int_{t_{2 m_{0}}}^{t_{2 m+1}} 2\left|\hat{\xi}_{1}^{\top} \tilde{\theta}^{\kappa}(\tau)\right|^{2}-\mu_{4}\left|\hat{\xi}_{1}^{\top} \tilde{\theta}^{2}(\tau)\right|^{2} d \tau$ where $\mu_{4}:=\frac{1}{2}-3 \hat{\varepsilon}^{2}(\varepsilon) \delta_{3}>0$ for sufficient small $\varepsilon$. As shown before, for $t>t_{2 m_{0}}$ the upper bound of $\left\|\tilde{\theta}^{\kappa}(t)\right\|^{2}$ depends on $\varepsilon$, whereas the lower bound of $\left\|\tilde{\theta}^{2}(t)\right\|^{2}$ is independent on $\varepsilon$. Consequently, if $\varepsilon$ is small enough then

$$
\tilde{J}\left(t_{2 m+1}\right) \leq \tilde{J}\left(t_{2 m_{0}}\right)-\beta \int_{t_{2 m_{0}}}^{t_{2 m+1}} \mu_{5} \hat{\xi}_{1}^{\top} \hat{\xi}_{1} d \tau
$$

for some positive constant $\mu_{5}$. Using (56), it follows that

$$
\tilde{J}\left(t_{2 m+1}\right) \leq \tilde{J}\left(t_{2 m_{0}}\right)-\left(m-m_{0}\right) \beta \mu_{3} \mu_{5} h .
$$

Consequently, there exists an integer $\bar{m}>m_{0}$ such that $\tilde{J}\left(t_{2 \bar{m}+1}\right) \leq-h$. Recalling the hysteresis switching logic (36), inequality (62) violates the assumption $\kappa \notin\{1,2\}$, hence the controller $\kappa$ participates in the non-terminating switching sequence. This completes the proof.

\section{G. Proof of Lemma 5.3}

Since the system is globally Lipschitz uniformly in $t$, boundedness of the signal $m_{i}(\cdot), i \in \mathcal{Q}$ on the finite time interval $\left[0, T_{s}\right]$ is automatically satisfied, where $T_{s}$ is defined in Proposition 5.1. Let $\sigma$ denote the label of the active controller after the switching has stopped, that is, $\sigma(t)=\sigma$, $\sigma \in \mathcal{Q}$, for all $t \geq T_{s}$. Setting $\epsilon:=\tilde{y}^{\sigma} / m_{\sigma}^{2}$ yields $\epsilon m_{\sigma} \in \mathcal{L}_{\infty}$. The properties of the $\mathcal{L}_{2 \delta}$ norm $^{3}$ are used to establish boundedness of $\hat{\zeta}_{o}^{\sigma}(\cdot)$. Rewrite the $\hat{\zeta}_{o}^{\sigma}$-subsystem as

$$
\dot{\hat{\zeta}}_{o}^{\sigma}=\left[S-\varepsilon \hat{\boldsymbol{\theta}}^{\sigma} \hat{\boldsymbol{\theta}}^{\sigma \top}\right] \hat{\zeta}_{o}^{\sigma}-\varepsilon \Gamma^{\top} \epsilon m_{\sigma}^{2}
$$

and consider the Lyapunov function candidate $W_{4}\left(t, \hat{\zeta}_{o}^{\sigma}\right):=$ $\hat{\zeta}_{o}^{\sigma \top} P_{o}\left(\hat{\theta}^{\sigma}(t), \varepsilon\right) \hat{\zeta}_{o}^{\sigma}$. The derivative of $W_{4}(\cdot)$ reads as

$$
\begin{aligned}
\dot{W}_{4} & =-\varepsilon \hat{\zeta}_{o}^{\sigma \top} \mathcal{I}_{o}\left(\hat{\theta}^{\sigma} \varepsilon\right) \hat{\zeta}_{o}^{\sigma}+\frac{\partial \hat{\zeta}_{o}^{\sigma \top} P_{o} \hat{\zeta}_{o}^{\sigma}}{\partial \hat{\theta}^{\sigma}} \dot{\hat{\theta}}^{\sigma}+2 \varepsilon \hat{\zeta}_{o}^{\sigma \top} P_{o} \Gamma^{\top}\left(\epsilon m_{\sigma}^{2}\right) \\
& \leq-\varepsilon N \delta_{1}^{\prime 2}\left\|\hat{\zeta}_{o}^{\sigma}\right\|^{2}+\varepsilon^{3} \rho \ell_{1}\left\|\hat{\zeta}_{o}^{\sigma}\right\|^{2}+2 \varepsilon c_{3} \sqrt{N}\left\|\hat{\zeta}_{o}^{\sigma}\right\|\left|\epsilon m_{\sigma}^{2}\right| \\
& \leq-\varepsilon\left(N \delta_{1}^{\prime 2}-\varepsilon^{2} \rho \ell_{1}-\varepsilon N c_{3}^{2}\right)\left\|\hat{\zeta}_{o}^{\sigma}\right\|^{2}+\left|\epsilon m_{\sigma}^{2}\right|^{2}
\end{aligned}
$$

for all $\left(\hat{\theta}_{n}^{\sigma}(\cdot), \varepsilon\right) \in \Theta_{\mathcal{R}(\sigma, n)} \times(0, \bar{\varepsilon}]$, where $\delta_{1}^{\prime}=\frac{\delta_{1}}{2}$. Letting $\varepsilon_{2}^{\star}=\min \left\{\bar{\varepsilon}, \frac{N \delta_{1}^{\prime 2}}{\bar{\varepsilon} \rho \ell_{1}+c_{3}^{2} N}\right\}$, one obtains $N \delta_{1}^{\prime 2}-\varepsilon^{2} \rho \ell_{1}-$ $\varepsilon N c_{3}^{2}:=\lambda_{4}(\varepsilon)>0$ for all $\varepsilon \in\left(0, \varepsilon_{2}^{\star}\right)$. Consequently

$\dot{W}_{4} \leq-\varepsilon \lambda_{4}(\varepsilon)\left\|\hat{\zeta}_{o}^{\sigma}\right\|^{2}+\left|\epsilon m_{\sigma}^{2}\right|^{2} \leq-\frac{\varepsilon \lambda_{4}(\varepsilon)}{c_{2}} W_{4}+\left|\epsilon m_{\sigma}^{2}\right|^{2}$

${ }^{3}$ Recall that, in this case, $\left\|x_{t}\right\|_{2 \delta}:=\left(\int_{T_{s}}^{t} e^{-\delta(t-\tau)} x^{\top}(\tau) x(\tau) d \tau\right)^{\frac{1}{2}}$ where we made use of Lemma 3.1. Integrating both sides of (63) and applying Lemma 3.1 yield, for all $t \in\left[T_{s}, \infty\right)$,

$$
\begin{aligned}
c_{1}\left\|\hat{\zeta}_{o}^{\sigma}(t)\right\|^{2} \leq c_{2} e^{-\alpha_{0}\left(t-T_{s}\right)}\left\|\hat{\zeta}_{o}^{\sigma}\left(T_{s}\right)\right\|^{2} & \\
& +\int_{T_{s}}^{t} e^{-\alpha_{0}(t-\tau)}\left(\epsilon m_{\sigma}^{2}\right)^{2}(\tau) d \tau
\end{aligned}
$$

where $\alpha_{0}:=\frac{\varepsilon \lambda_{4}(\varepsilon)}{c_{2}}$. Taking the square root and applying the triangle inequality, one obtains

$$
\left\|\hat{\zeta}_{o}^{\sigma}(t)\right\| \leq \kappa_{1}\left\|\epsilon m_{\sigma t}^{2}\right\|_{2 \alpha_{0}}+\kappa_{0}(\varepsilon, t) \quad \forall t \in\left[T_{s},+\infty\right)
$$

where $\kappa_{1}:=\frac{1}{\sqrt{c_{1}}}$ and $\kappa_{0}(\varepsilon):=\sqrt{\frac{c_{2}}{c_{1}}} e^{\frac{\alpha_{0}}{2}} T_{s}\left\|\hat{\zeta}_{o}^{\sigma}\left(T_{s}\right)\right\|$. Exploiting the triangle inequality property of the $L_{2 \delta}$ norm, it follows that $\left\|\hat{\zeta}_{o t}^{\sigma}\right\|_{2 \delta} \leq \kappa_{1}\left\|Q_{t}\right\|_{2 \delta}+\left\|\kappa_{0}(\varepsilon, \cdot)_{t}\right\|_{2 \delta}$, where

$$
\left\|Q_{t}\right\|_{2 \delta}=\left[\int_{T_{s}}^{t} e^{-\delta(t-\tau)}\left(\int_{T_{s}}^{\tau} e^{-\frac{\alpha_{0}}{2}(\tau-s)}\left|\epsilon m_{\sigma}^{2}\right|(s) d s\right)^{2} d \tau\right]^{\frac{1}{2}}
$$

Using Schwartz inequality [20, Lemma 3.2.1], one obtains for any $\delta \in\left[0, \bar{\delta}_{1}\right)$ where $0<\bar{\delta}_{1}<\alpha_{0}$

$$
\begin{aligned}
& \left(\int_{T_{s}}^{\tau} e^{-\frac{\alpha_{0}}{2}(\tau-s)}\left|\epsilon m_{\sigma}^{2}\right|(s) d s\right)^{2} \\
& =\left(\int_{T_{s}}^{\tau} e^{-\left(\frac{\alpha_{0}}{2}-\frac{\bar{\delta}_{1}}{2}\right)(\tau-s)} e^{-\frac{\bar{\delta}_{1}}{2}(\tau-s)}\left|\epsilon m_{\sigma}^{2}\right|(s) d s\right)^{2} \\
& \leq \kappa_{1}^{\prime} \int_{T_{s}}^{\tau} e^{-\bar{\delta}_{1}(\tau-s)}\left(\epsilon m_{\sigma}^{2}\right)^{2}(s) d s
\end{aligned}
$$

with $\kappa_{1}^{\prime}:=\frac{1}{\alpha_{0}-\bar{\delta}_{1}}$, yelding the upper bound $\left\|Q_{t}\right\|_{2 \delta} \leq$ $\sqrt{\kappa_{1}^{\prime}}\left(\int_{T_{s}}^{t} e^{-\delta(t-\tau)} \int_{T_{s}}^{\tau} e^{-\bar{\delta}_{1}(\tau-s)}\left(\epsilon m_{\sigma}^{2}\right)^{2}(s) d s d \tau\right)^{\frac{1}{2}}$. Interchanging the sequence of the integration, one obtains

$$
\begin{aligned}
& \left\|Q_{t}\right\|_{2 \delta} \leq \sqrt{\kappa_{1}^{\prime}}\left(\int_{T_{s}}^{t} e^{-\delta t+\bar{\delta}_{1} s}\left(\epsilon m_{\sigma}^{2}\right)^{2}(s) \int_{s}^{t} e^{-\left(\bar{\delta}_{1}-\delta\right) \tau} d \tau d s\right)^{\frac{1}{2}} \\
& \leq \kappa_{2}\left(\int_{T_{s}}^{t}\left(\epsilon m_{\sigma}^{2}\right)^{2}(s)\left[e^{-\delta(t-s)}-e^{-\bar{\delta}_{1}(t-s)}\right] d s\right)^{\frac{1}{2}}
\end{aligned}
$$

with $\kappa_{2}:=\frac{1}{\sqrt{\left(\alpha_{0}-\bar{\delta}_{1}\right)\left(\bar{\delta}_{1}-\delta\right)}}$. Since $\left\|\kappa_{0}(\varepsilon)\right\|_{2 \delta} \leq \kappa_{0}(\varepsilon) / \sqrt{\delta}$, then

$$
\left\|\hat{\zeta}_{o t}^{\sigma}\right\|_{2 \delta} \leq \kappa\left\|\epsilon m_{\sigma_{t}}^{2}\right\|_{2 \delta}+\frac{\kappa_{0}(\varepsilon)}{\sqrt{\delta}}
$$

for all $0<\delta<\bar{\delta}_{1}<\alpha_{0}$, where $\kappa:=\frac{1}{\sqrt{c_{1}\left(\alpha_{0}-\delta_{1}\right)\left(\bar{\delta}_{1}-\delta\right)}}$. Next, we show that $\left\|\hat{\xi}_{1}(t)\right\|,\left\|\xi_{2}(t)\right\|,\left\|\xi_{3}(t)\right\|,\|z(t)\|$ can be bounded in terms of $\left\|\hat{\zeta}_{o_{t}}^{\sigma}\right\|_{2 \delta}$. Recall that the $\hat{\xi}_{1}$-dynamics wth the active controller read as

$$
\dot{\hat{\xi}}_{1}=E_{\varepsilon}^{\top} \hat{\xi}_{1}-\varepsilon G \hat{\boldsymbol{\theta}}^{\sigma \top} \hat{\zeta}_{o}^{\sigma} .
$$

Since $E_{\varepsilon}$ is Hurwitz, there exists positive constants $\alpha_{1}, l_{1}$ satisfy $\left\|e^{E_{\varepsilon}^{\top}(t-\tau)}\right\| \leq l_{1} e^{-\alpha_{1}(t-\tau)}$. Then making use of the boundedness of the term $\varepsilon G \hat{\boldsymbol{\theta}}^{\top}$ and the properties of the $\mathcal{L}_{2 \delta}$ norm [20, Lemma 3.3.3], for any constant $\delta \in\left[0, \bar{\delta}_{2}\right)$ where $0<\bar{\delta}_{2}<2 \alpha_{1}$, it follows that $\left\|\hat{\xi}_{1}(t)\right\| \leq \bar{\kappa}\left\|\hat{\zeta}_{o t}^{\sigma}\right\|_{2 \delta}+\bar{\kappa}_{0}$ for some finite constants $\bar{\kappa}, \bar{\kappa}_{0}>0$. Similarly, for the subsystem (22) where $F_{\varepsilon}$ is constant and Hurwitz, one obtains

$$
\max \left\{\left\|\hat{\xi}_{2}(t)\right\|,\left\|\hat{\xi}_{3}(t)\right\|,\|z(t)\|\right\} \leq \bar{\kappa}\left\|\hat{\zeta}_{\text {ot }}^{\sigma}\right\|_{2 \delta}+\bar{\kappa}_{0}
$$


for any $\delta \in\left[0, \delta^{\star}\right)$ where $\delta^{\star}$ is a positive constant. Then the signal $m_{\sigma}^{2}$ satisfies

$$
\begin{aligned}
m_{\sigma}^{2} \leq 1+ & \left\|\hat{\xi}_{1}(t)\right\|^{2}+\left\|\tilde{\theta}^{\sigma \top}(t) \hat{\xi}_{1}(t)\right\|^{2}+\left\|\Gamma \xi_{2}(t)\right\|^{2} \\
& +\left\|\Gamma \xi_{3}(t)\right\|+\|C(\mu) z(t)\|^{2} \leq \bar{k}\left\|\hat{\zeta}_{o t}^{\sigma}\right\|_{2 \delta}^{2}+\bar{k}_{0}
\end{aligned}
$$

for some finite constants $\bar{k}, \bar{k}_{0}>0$. Letting $\bar{k}\left\|\hat{\zeta}_{o t}^{\sigma}\right\|_{2 \delta}^{2}+$ $\bar{k}_{0}:=m_{f}^{2}$, it follows that $m_{\sigma} \leq m_{f}$ and, due to (65),

$$
\begin{aligned}
m_{f}^{2} & \leq k\left\|\epsilon m_{\sigma t}^{2}\right\|_{2 \delta}^{2}+k_{0} \leq k\left\|m_{f} \epsilon m_{\sigma t}\right\|_{2 \delta}^{2}+k_{0} \\
& \leq k \int_{T_{s}}^{t} e^{-\delta(t-\tau)}\left(\epsilon m_{\sigma}\right)^{2}(\tau) m_{f}^{2}(\tau) d \tau+k_{0}
\end{aligned}
$$

for some finite constants $k, k_{0}>0$. Applying BellmanGronwall inequality [20, Lemma 3.3.7], one obtains

$$
m_{f}^{2}(t) \leq k_{0} e^{k \int_{T_{s}}^{t} e^{-\delta(t-\tau)}\left(\epsilon m_{\sigma}\right)^{2}(\tau) d \tau} .
$$

Since $\epsilon m_{\sigma} \in \mathcal{L}_{\infty}$ and $\left\|\epsilon m_{\sigma}\right\|_{\infty} \leq 1$

$$
\int_{T_{s}}^{t} e^{-\delta(t-\tau)}\left(\epsilon m_{\sigma}\right)^{2}(\tau) d \tau \leq\left\|\epsilon m_{\sigma}\right\|_{\infty}^{2} \int_{T_{s}}^{t} e^{-\delta(t-\tau)} d \tau \leq \frac{1}{\delta}
$$

then we have $m_{f}(t) \leq \sqrt{k_{0}} e^{\frac{k}{2 \delta}}$, therefore $m_{f}$ and $m_{\sigma} \in$ $\mathcal{L}_{\infty}$. For the inactive controller $i, i \in \mathcal{Q}, i \neq \sigma$, the signal $m_{i}^{2}$ admits the following upper bound

$$
\begin{aligned}
& m_{i}^{2}=1+\left\|\hat{\xi}_{1}\right\|^{2}+\left|\hat{\xi}_{1}^{\top} \tilde{\theta}^{i}+y_{1}\right|^{2} \\
& =1+\left\|\hat{\xi}_{1}\right\|^{2}+\left|\hat{\xi}_{1}^{\top}\left(\tilde{\theta}^{\sigma}+\hat{\theta}^{i}-\hat{\theta}^{\sigma}\right)+y_{1}\right|^{2} \\
& \leq 1+\left\|\hat{\xi}_{1}\right\|^{2}+\left|\tilde{y}^{\sigma}\right|^{2}+\left|\hat{\xi}_{1}^{\top}\left(\hat{\theta}^{i}-\hat{\theta}^{\sigma}\right)\right|^{2} \leq m_{\sigma}^{2}+\left|\hat{\xi}_{1}^{\top}\left(\hat{\theta}^{i}-\hat{\theta}^{\sigma}\right)\right|^{2}
\end{aligned}
$$

Since $m_{\sigma}, \hat{\xi}_{1}, \hat{\theta}^{i}-\hat{\theta}^{\sigma} \in \mathcal{L}_{\infty}, m_{i} \in \mathcal{L}_{\infty}$ as well.

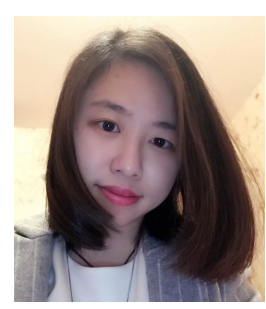

Yang Wang received the B. Eng. in electrical and electronic engineering in 2013 from Tongji University, Shanghai, China. In 2014, she received the M.Sc. degree in control systems from Imperial College London, U.K, where she has obtained her Ph.D. degree in 2019. Her research interests include output regulation, estimation and identification of periodic signal and its applications.

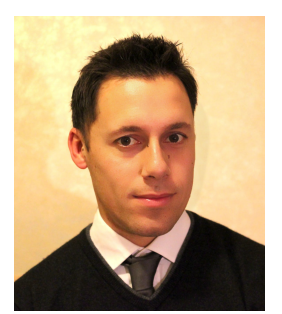

Gilberto Pin received the Laurea (M.Sc.) degree in Electrical Engineering (with honors) and the $\mathrm{Ph} . \mathrm{D}$. in Information Engineering from the University of Trieste, Italy, in 2005 and 2009, respectively. From 2009 to 2012 he was an Automation Engineer at Danieli Automation S.p.A., Italy. From 2013 to 2015 he has been a Control Systems Engineer at the R\&D Dept. of Electrolux Professional S.p.A., Italy. Since 2015, he is an Energy Conversion Engineer at the Global Connectivity \& Technology Center of Electrolux Major Appliances S.p.A., Italy. He is co-recipient of the IFAC Best Application Paper Prize of the Journal of Process Control, Elsevier, for the three-year period 2011-2013 and of the Electrolux Invention Award in 2017. His research interests include nonlinear model predictive control, adaptive control and industrial application of advanced control techniques. Since 2012 he serves as Associate Editor of the Conference Editorial Board of the IEEE Control Systems Society and of the IEEE Trans. on Control Systems Technology.

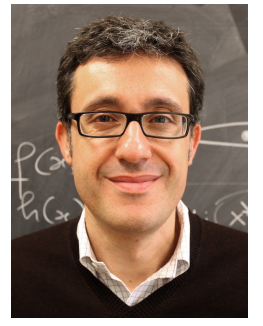

Andrea Serrani received the Laurea (B.Eng.) degree in Electrical Engineering, summa cum laude, from the University of Ancona, Italy, in 1993, and the Ph.D. degree in Artificial Intelligence Systems from the same institution in 1997. From 1994 to 1999, he was a Fulbright Fellow at Washington University in St. Louis, MO, where he obtained the M.S. and D.Sc. degrees in Systems Science and Mathematics in 1996 and 2000, respectively. Since 2002, he has been with the Department of Electrical and Computer Engineering of The Ohio State University, where he is currently a Professor and Associate Chair. He has held visiting positions at the Universities of Bologna and Padua, Italy, and multiple summer faculty fellowships at AFRL. The research activity of Prof. Serrani lies at the intersection of methodological aspects of nonlinear, adaptive and geometric control theory with applications in aerospace and marine systems, fluidic systems, robotics and automotive engineering. His work has been supported by AFRL, NSF, Ford Motor Co. and NASA, among others. Prof. Serrani has authored or co-authored more than 150 articles in journals, proceedings of international conferences and book chapters, and is the co-author of the book Robust Autonomous Guidance: An Internal Model Approach published by Springer-Verlag. Prof. Serrani was a Distinguished Lecturer of the IEEE CSS. Prof. Serrani is the Editor-in-Chief of the IEEE Trans. on Control Systems Technology and a past Associate Editor for the same journal (2010-2016), Automatica (2008-2014) and the Int. Journal of Robust and Nonlinear Control (2006-2014). He serves on the Conference Editorial Boards of IEEE CSS and IFAC, served as Program Chair for the 2019 ACC, and will serve as General Co-chair for the 2022 CDC.

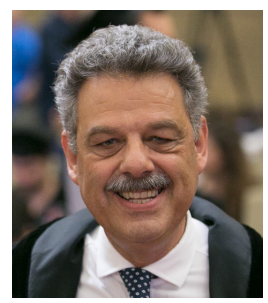

Thomas Parisini received the Ph.D. degree in Electronic Engineering and Computer Science in 1993 from the University of Genoa. He was with Politecnico di Milano and since 2010 he holds the Chair of Industrial Control and is Director of Research at Imperial College London. He is a Deputy Director of the KIOS Research and Innovation Centre of Excellence, University of Cyprus. Since 2001 he is also Danieli Endowed Chair of Automation Engineering with University of Trieste. In 2009-2012 he was Deputy Rector of University of Trieste. In 2018, he received an Honorary Doctorate from University of Aalborg, Denmark. He authored or co-authored more than 300 research papers in archival journals, book chapters, and international conference proceedings. His more recent research interests include monitoring, diagnosis, control and cyber-security of large-scale interconnected nonlinear systems with applications in smart grids, power electronics and industrial process control. He is a co-recipient of the IFAC Best Application Paper Prize of the Journal of Process Control, Elsevier, for the three-year period 2011-2013 and of the 2004 Outstanding Paper Award of the IEEE Trans. on Neural Networks. In 2016 he was awarded as Principal Investigator at Imperial of the H2020 European Union flagship Teaming Project KIOS Research and Innovation Centre of Excellence led by University of Cyprus. In 2012 he was awarded an ABB Research Grant dealing with energyautonomous sensor networks for self-monitoring industrial environments. Thomas Parisini currently serves as Vice-President for Publications Activities and has been recently elected as the 2020 President-Elect of the IEEE Control Systems Society. During 2009-2016 he was the Editor-in-Chief of the IEEE Trans. on Control Systems Technology and since 2018 he is the Editor in Chief of the European Journal of Control. Since 2017, he is also Editor for Control Applications of Automatica. Among other activities, he was the General Co-Chair of the 2013 IEEE Conference on Decision and Control. Prof. Parisini is a Fellow of the IEEE and of the IFAC. 